\title{
Further investigations into the preparation and [4+2] cycloaddition reactions of vinyl norcaradiene derivatives
}

\author{
Lisa A. Buttle, ${ }^{a}$ Jonathan C. Morris, $^{b}$ and Lewis N. Mander*a \\ ${ }^{a}$ Research School of Chemistry, Institute of Advanced Studies, Australian National University, \\ Canberra, A.C.T. 0200, Australia, and ${ }^{b}$ Department of Chemistry, University of Canterbury, \\ Christchurch, New Zealand \\ E-mail: mander@rsc.anu.edu.au
}

Dedicated to Professor Keiichiro Fukumoto on the occasion of his $\mathbf{7 0}^{\text {th }}$ birthday

(received 30 May 03; accepted 22 July 03; published on the web 04 Aug 03)

\begin{abstract}
Norcaradiene derivatives derived from the copper-catalyzed intramolecular cyclopropanation reactions of 2-(5-vinyl-1,2,3,4-tetrahydronaphthalene) diazomethyl ketones have been prepared and submitted to Diels-Alder reactions with a range of dienophiles, including methyl acrylate, methyl 2-chloroacrylate, maleic anhydride and citraconic anhydride. The cycloaddition reactions gave better yields and were more selective when the vinyl norcaradienes were based on 8methoxynaphthyl diazoketones.
\end{abstract}

Keywords: Diazoketone, norcaradiene, Diels-Alder, cycloaddition

\section{Introduction}

We recently described a new and efficient strategy for the synthesis of the gibberellin-derived antheridiogens 5 and $\mathbf{6}$ as outlined in Scheme 1. ${ }^{1}$ Of special note is the cyclopropanation step followed by the pivotal in situ cycloaddition ${ }^{2}$ to allow the stereocontrolled elaboration of the key intermediate 3, an exceptionally versatile intermediate for the preparation of a wide range of tetracyclic diterpenoids. ${ }^{3}$ This sequence, however, was the culmination of extensive explorations with several analogues of both the diene and dienophile participants. In this paper, we disclose the details of those investigations. 

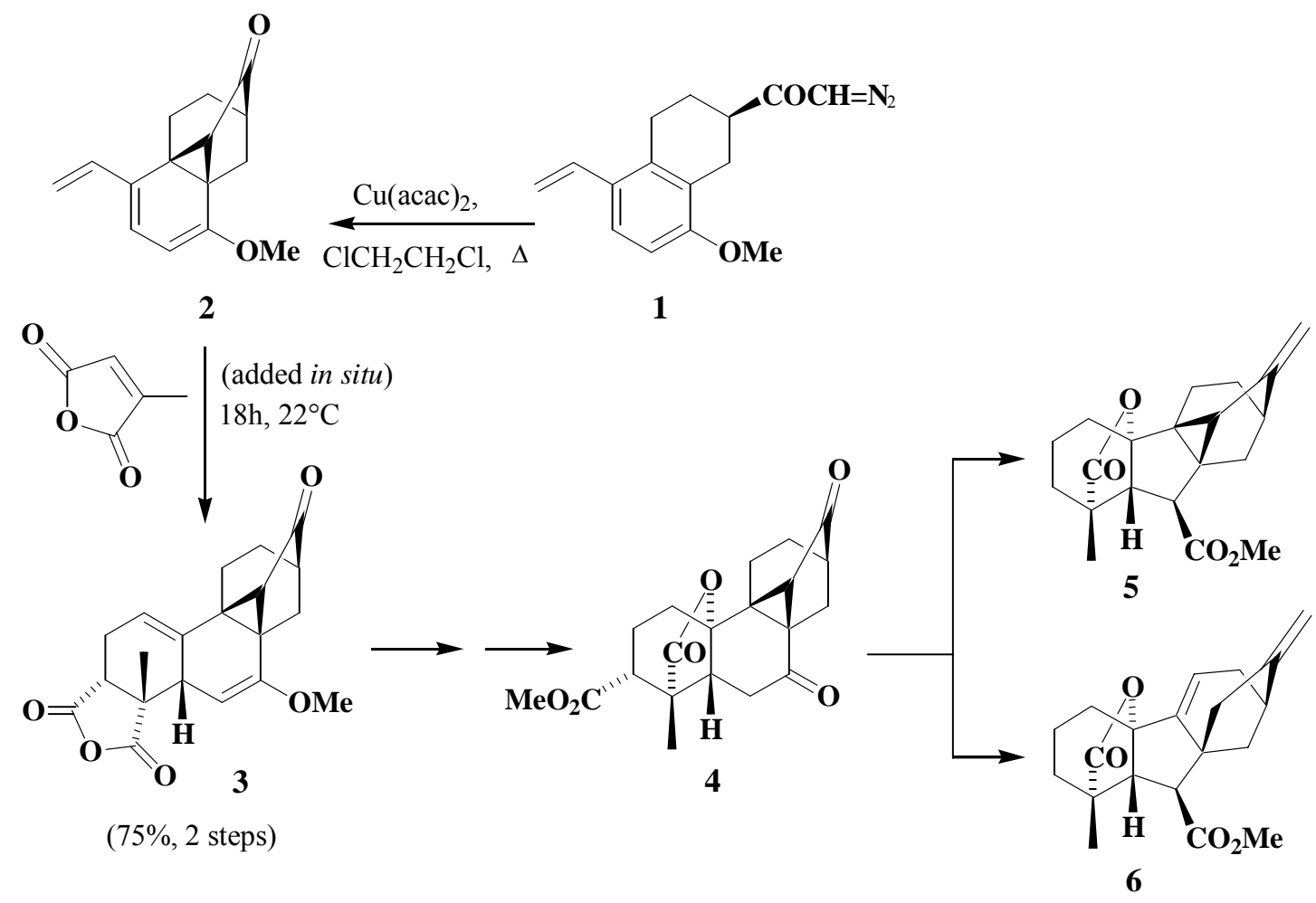

\section{Scheme 1}

\section{Results and Discussion}

Our early attempts to prepare the styrene diazoketone 1 using standard procedures (diazomethane treatment of the acid chloride) $)^{4}$ were thwarted by the polymerization of the styrene moiety on exposure to adventitious acid generated during formation of the acid chloride. We therefore decided to approach the preparation of the cyclopropyl ketone 2 via the analogous aldehyde, as outlined in Scheme 2.

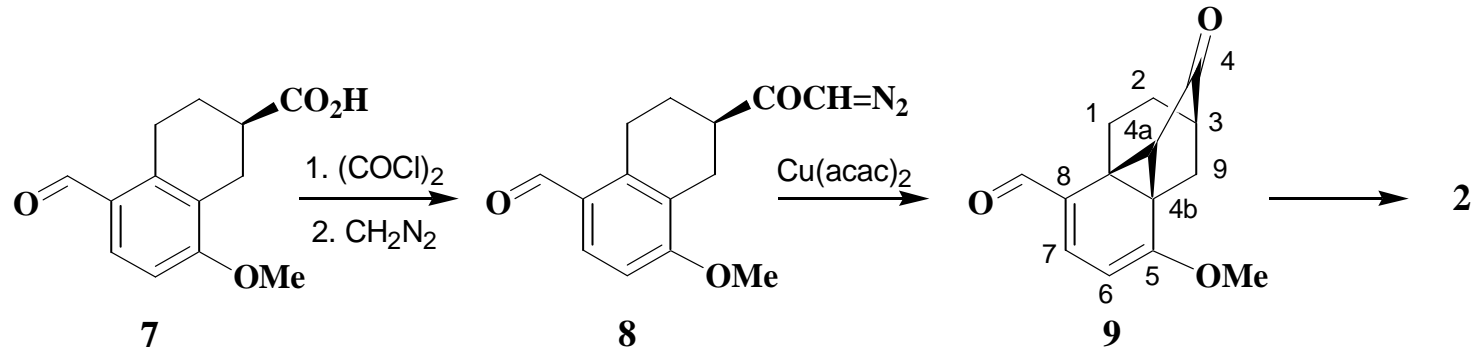

\section{Scheme 2}


Treatment of the acid $7^{5}$ with oxalyl chloride and a catalytic amount of DMF in dichloromethane (Scheme 3) resulted in a single compound, but ${ }^{1} \mathrm{H}$ - and ${ }^{13} \mathrm{C}$ NMR spectroscopy revealed the disappearance of the aldehyde functionality. The presence of an acid chloride moiety was confirmed by an absorption at $1790 \mathrm{~cm}^{-1}$ in the infrared spectrum, while the ${ }^{1} \mathrm{H}$ NMR spectrum was essentially the same as that of the acid, with the exception of a singlet resonating at $6.91 \mathrm{ppm}$ in lieu of the aldehyde proton; the ${ }^{13} \mathrm{C}$ APT spectrum showed a methine resonance at $69.6 \mathrm{ppm}$. We concluded, therefore, that the carboxaldehye function had been replaced by a dichloromethyl group, and pressed on with the elaboration of the diazoketone 11 which, when dissolved in a mixture of triethylamine and aqueous acetone, afforded the desired diazoketone 8 in $63 \%$ overall yield. The presence of the diazoketone function was confirmed by the infrared absorption at $2105 \mathrm{~cm}^{-1}$ and a broad signal at $5.45 \mathrm{ppm}$ in the ${ }^{1} \mathrm{H}$ NMR spectrum. The broadness of the peak from the proton attached to the diazoketone moiety has been ascribed to free rotation about the $\mathrm{C}-\mathrm{C}$ bond being hindered as a result of the interaction of the $\pi$ electrons on the $\alpha$-carbon with the $\pi$-system of the carbonyl group, resulting in an equilibrium mixture of cis- and trans- rotamers. ${ }^{6}$ Finally, the diazoketone 8 was treated with catalytic $\mathrm{Cu}(\mathrm{acac})_{2}$ in dry 1,2-dichloroethane at reflux, to give the formylnorcaradiene 9 in $65 \%$ yield.

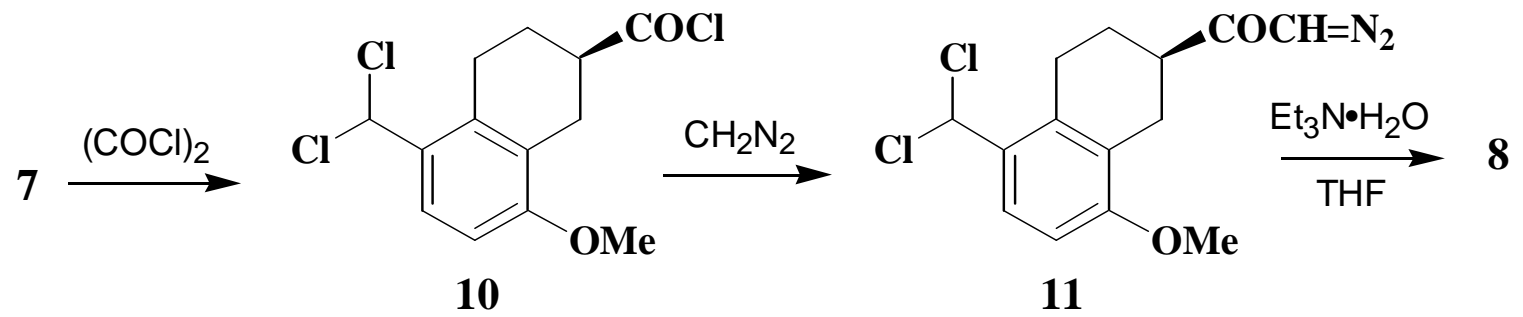

\section{Scheme 3}

Attempts to methylenate the aldehyde function in the norcaradiene 9 were initially fruitless, with the Wittig reaction, Lombardo-Oshima reaction, ${ }^{7}$ and treatment with zirconium cyclopentadiene dichloride/diiodomethane ${ }^{8}$ all resulting in rapid decomposition of the substrate. Limited success was achieved with the Peterson reagent, TMS- $\mathrm{CH}_{2} \mathrm{Li}^{9}{ }^{9}$ however (Scheme 4). Addition of three molar equivalents at $0{ }^{\circ} \mathrm{C}$, followed by $\mathrm{KH}$, resulted in the formation of two unstable compounds in low yield. One was identified as the desired triene $\mathbf{1 3}$ (from the ${ }^{1} \mathrm{H}$ NMR resonances typical of the vinyl group), while the other product appeared to be the enone 14. ${ }^{1} \mathrm{H}$ NMR signals for two distinct TMS groups and a triplet at $6.05 \mathrm{ppm}$ were considered diagnostic for the allyl-silane while a pair of doublets $(J=9.7 \mathrm{~Hz})$ at 6.05 and $7.00 \mathrm{ppm}$ was consistent with the $\alpha, \beta$-enone moiety. The overall yield of these products was only $21 \%$ and this approach was accordingly abandoned. 


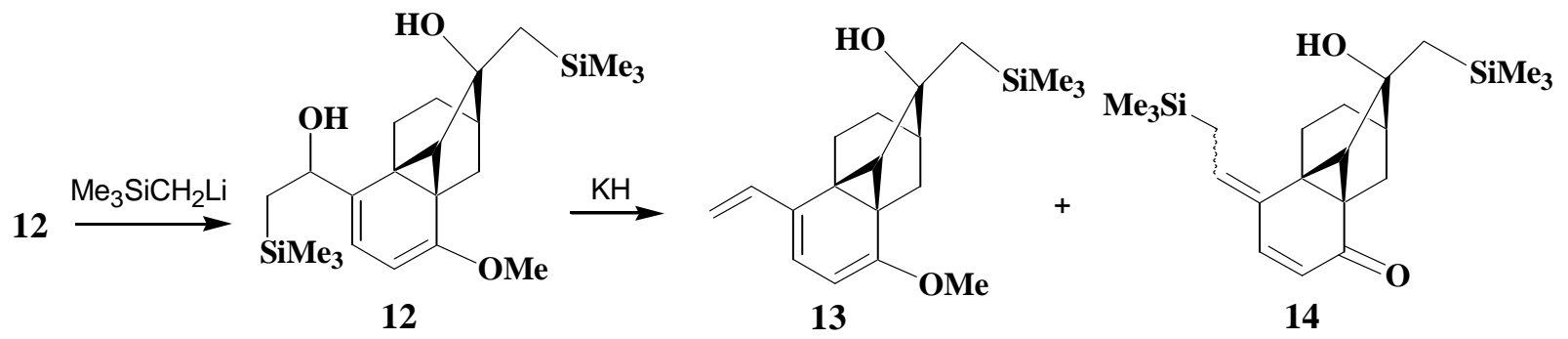

\section{Scheme 4}

Our next attempt to deal with the problem of the acid-promoted polymerization of the styrene functionality is outlined in Scheme 5. This approach involved the attachment of an electronwithdrawing group to the vinyl substituent. A nitrile group appeared particularly attractive, since nitriles, inter alia, can be converted into ketones via hydroperoxy derivatives ${ }^{10}$ and would allow for straightforward functionalization of the A-ring subsequently. The cyanovinyl acid $\mathbf{1 5}$ was obtained as a 3:1 mixture of $E$ - and $Z$ - isomers from the formyl acid 7 in $90 \%$ yield using a Horner-Wadsworth-Emmons reaction. ${ }^{11}$ Even though the Z-isomer was not expected to react under the Diels-Alder conditions, ${ }^{12}$ the desired $E$ isomer was easily separated by recrystallization from methanol. The large trans coupling $(J=16.4 \mathrm{~Hz})$ of the alkene protons was compelling evidence for the stereochemistry of the double bond, while IR absorption at $2225 \mathrm{~cm}^{-1}$ and a ${ }^{13} \mathrm{C}$ NMR resonance at $118.8 \mathrm{ppm}$, confirmed that the nitrile group was present. When the E-cyanovinyl acid 15 was added to oxalyl chloride and a catalytic quantity of DMF in dichloromethane, a smooth reaction took place. Treatment of the crude product with an ethereal solution of diazomethane gave the diazoketone $\mathbf{1 6}$ as a yellow crystalline solid in $71 \%$ yield. Then cyclopropanation, under the same conditions as those used for the formyl diazoketone 8, gave the E-cyanovinyl norcaradiene $\mathbf{1 7}$ in $60 \%$ yield. To test the use of the Diels-Alder reaction for the addition of the A-ring, norcaradiene $\mathbf{1 7}$ and maleic anhydride (carefully sublimed to remove any traces of maleic acid) were dissolved in benzene and the solution heated to $80{ }^{\circ} \mathrm{C}$. After 48 hours the starting material had been totally consumed. Isolation and recrystallization of the residue from acetone afforded an especially insoluble white solid in $88 \%$ yield, with ${ }^{1} \mathrm{H}$ NMR spectroscopy indicating the presence of a single diastereomer presumed to be the endo adduct 18 resulting from addition to the lower face of triene $\mathbf{1 7}$ (cf., ref. 1). The small vicinal coupling constant between H-8 and H-9 indicated that these hydrogens were on the same side of the molecule (i.e., cis), which confirmed that the endo adduct had been formed. In view of subsequent developments, ${ }^{1}$ we have not yet pursued this chemistry further, but clearly, it has considerable potential. 


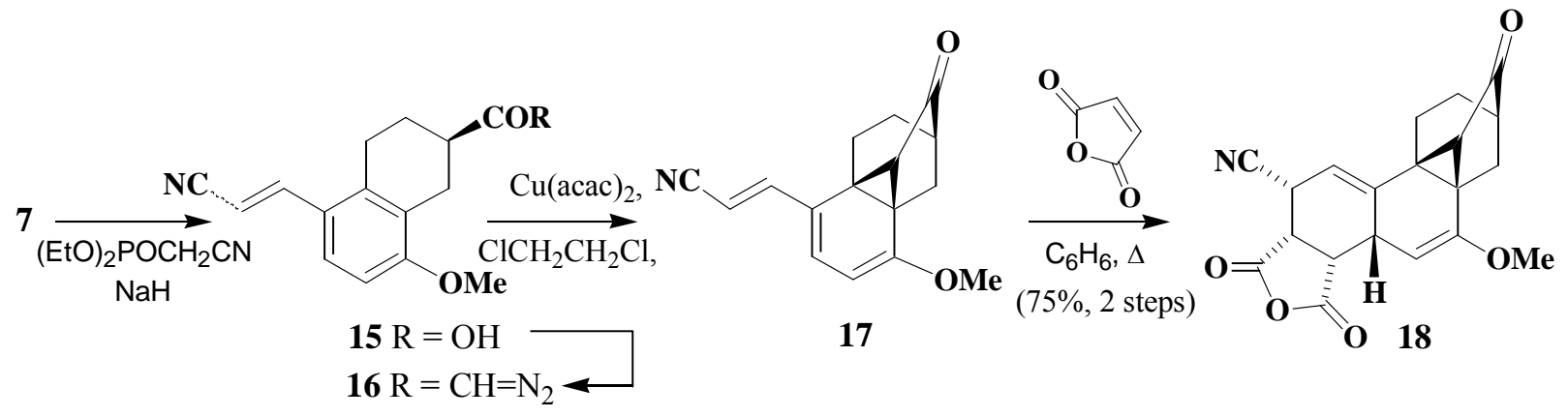

\section{Scheme 5}

As an alternative to deactivating the styrene functionality in the diazoketone precursors by the addition of an electron-withdrawing substituent, we decided to explore the prospects of utilizing the des-methoxy analogue of $\mathbf{1}$, in the expectation that the reduction in electron density would afford more stable intermediates. The required starting material was prepared by means of a Stille coupling ${ }^{13}$ (Scheme 6) and, indeed, the derived intermediates proved to be considerably more stable, allowing smooth elaboration of the vinyl norcaradiene 22 in good overall yield. In the meantime, we had solved the problem of preparing diazoketone $\mathbf{1}$ (as recorded elsewhere) ${ }^{1,2}$ and so had in hand a good supply of both norcaradienes 2 and 22, which allowed us to evaluate the suitability of various dienophiles for the addition of the A-ring. The results are summarized in Scheme 7, and include results from other studies for completeness. It is apparent from these outcomes that the more electron rich triene 2 affords superior yields and stereo-control, and so our efforts to date have been focused on its use. Nevertheless, the present disclosures allow us to define the scope and limitations of this strategy for the rapid assembly of kaurenoid- and related natural products. Minor variations in the substitution pattern of the initial substrates could allow access to atisane, stemarane and thyrsiflorane skeletons as well, ${ }^{3}$ and although the present study has been conducted with racemates, good methodology for the enantioselective synthesis of tetralin carboxylic acids is available. ${ }^{14}$

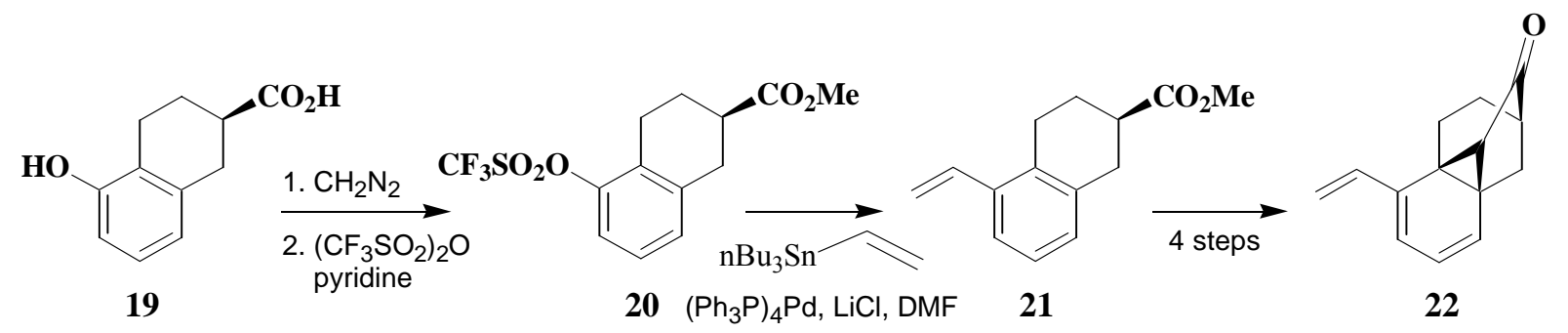

\section{Scheme 6}



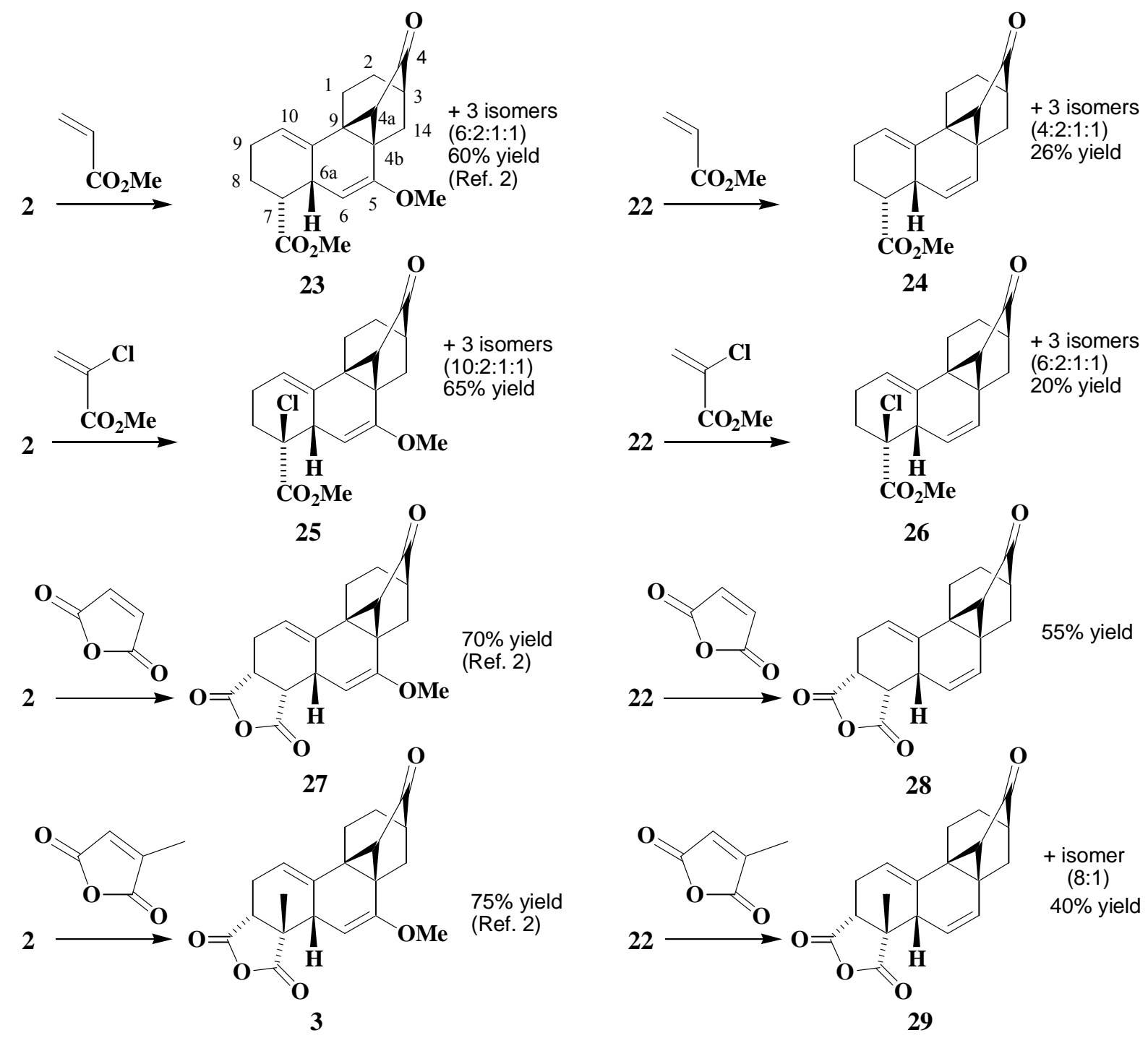

Scheme 7

\section{Experimental Section}

General Procedures. Infrared spectra $\left(v_{\max }, \mathrm{cm}^{-1}\right)$ were recorded on a Perkin-Elmer 1800 Fourier Transform Infrared spectrometer or a Perkin-Elmer 683 Infrared Spectrometer using $\mathrm{NaCl}$ or KI plates. NMR spectra were recorded on Varian Gemini $300 \mathrm{MHz}$ or Varian VXR 500 $\mathrm{MHz}$ instruments. Chemical shifts are reported in parts per million $(\delta \mathrm{ppm})$. For proton spectra recorded in chloroform, the residual peak of $\mathrm{CHCl}_{3}$ was used as the internal reference $(7.26 \mathrm{ppm})$ while the central peak of $\mathrm{CDCl}_{3}(77.0 \mathrm{ppm})$ was used as the reference for ${ }^{13} \mathrm{C}$ spectra. Multiplicities are abbreviated: $\mathrm{s}=$ singlet, $\mathrm{d}=$ doublet, $\mathrm{t}=$ triplet, $\mathrm{q}=$ quartet, $\mathrm{m}=$ multiplet, $\mathrm{br}=$ broad. Coupling constants are recorded in Hertz $(\mathrm{Hz})$. Distortionless enhancement by 
polarization transfer (DEPT) and attached proton test (APT) experiments were used in the assignment of carbon spectra. Mass spectra (EIMS, $70 \mathrm{eV}$ ) were recorded on a VG Micromass 7070F double focusing mass spectrometer. Analytical thin layer chromatography (TLC) used Merck aluminum backed TLC sheets with silica gel 60 F254, or Merck glass-backed TLC plates coated with $0.2 \mathrm{~mm}$ thick silica gel GF254. The developed plates were visualized under shortwave UV light and exposed to an ammonium molybdate dip with heat. All flash chromatography used the flash technique as reported by Still ${ }^{15}$ using Merck Kieselgel 60 and analytical reagent (AR) grade solvents as indicated. MPLC was conducted using a CfG Prominent Duramat ${ }^{\circledR}$ pump, a Waters Associates Differential Refractometer and Merck Lobar ${ }^{\circledR}$ Fertigsäule Größe LiChroprep ${ }^{\circledR}$ Si60 (40-63 $\left.\mu \mathrm{m}\right)$ columns.

(2-RS)-Diazomethyl 5-formyl-8-methoxy-1,2,3,4-tetrahydronaphthalen-2-yl ketone, (8). A solution of the acid $7^{5}(2 \mathrm{~g}, 8.55 \mathrm{mmol})$ in dichloromethane $(150 \mathrm{~mL})$ was added dropwise to a solution of oxalyl chloride $(5 \mathrm{~mL})$ and dichloromethane $(15 \mathrm{~mL})$ at room temperature under a nitrogen atmosphere. One drop of dry DMF was added (evolution of gas) and the resultant solution stirred for $14 \mathrm{~h}$. The volatile components were removed in vacuo and the procedure repeated twice more to afford the dichloromethyl acid chloride $\mathbf{1 0}$ as a yellow oil (100\%). IR $\left(\mathrm{CHCl}_{3}\right)$ 2950, 1790, 1590, $1480 \mathrm{~cm}^{-1} .{ }^{1} \mathrm{H}$ NMR (300 MHz, $\left.\mathrm{CDCl}_{3}\right) \delta 2.00(1 \mathrm{H}, \mathrm{m}, \mathrm{H}-3), 2.42$ (1H, m, H-3), 2.79-3.00 (2H, m, H-2 and H-1), 3.05-3.30 (3H, m, H-1, H-4 × 2), $3.88(3 \mathrm{H}, \mathrm{s}$, OMe), $6.81(1 \mathrm{H}, \mathrm{d}, J=8.7 \mathrm{~Hz}, \mathrm{H}-7), 6.91\left(1 \mathrm{H}, \mathrm{s}, \mathrm{CHCl}_{2}\right), 7.69(1 \mathrm{H}, \mathrm{d}, J=8.7 \mathrm{~Hz}, \mathrm{H}-6) .{ }^{13} \mathrm{C}$ NMR (75 MHz, CDCl $\left.)_{3}\right) \delta 24.4\left(\mathrm{CH}_{2}\right), 24.9\left(\mathrm{CH}_{2}\right), 25.9\left(\mathrm{CH}_{2}\right), 30.5(\mathrm{C}-2), 55.4(\mathrm{OMe}), 69.6$ $\left(\mathrm{CHCl}_{2}\right), 107.5$ (C-7), 122.8 (C-8a), 126.3 (C-6), 129.6 (C-4a), 132.7 (C-5), 158.0 (C-8), 176.4 $(\boldsymbol{C O C l})$.

After $1 \mathrm{~h}$ under high vacuum, to remove any traces of $\mathrm{HCl}$, the oil was dissolved in dichloromethane $(100 \mathrm{~mL})$ and added dropwise to fresh ethereal diazomethane $(0.4 \mathrm{M}, 5$ equiv.) at $-20{ }^{\circ} \mathrm{C}$ under nitrogen. After stirring for $15 \mathrm{~h}$, the yellow solution was filtered through Celite in a well-ventilated fume-hood. The solvent was removed in vacuo to afford the dichloromethyl diazoketone 11 as an orange oil (100\%). IR $\left(\mathrm{CHCl}_{3}\right) 2970,2105,1640 \mathrm{~cm}^{-1}$. ${ }^{1} \mathrm{H}$ NMR $(300$ $\mathrm{MHz}_{\mathrm{CDCl}}$ ) $\delta 1.80(1 \mathrm{H}, \mathrm{m}, \mathrm{H} 3), 2.15(1 \mathrm{H}, \mathrm{m}, \mathrm{H}-3), 2.60(1 \mathrm{H}, \mathrm{m}, \mathrm{H}-2), 2.55-3.15$ (4H, m, H-1 and $\mathrm{H}-4), 3.82(3 \mathrm{H}, \mathrm{s}, \mathrm{OMe}), 5.42\left(1 \mathrm{H}\right.$, br s, COCHN $\left.\mathrm{N}_{2}\right), 6.76(1 \mathrm{H}, \mathrm{d}, J=8.7 \mathrm{~Hz}, \mathrm{H}-7), 6.90(1 \mathrm{H}$, $\left.\mathrm{s}, \mathrm{CHCl}_{2}\right), 7.66(1 \mathrm{H}, \mathrm{d}, J=8.7 \mathrm{~Hz}, \mathrm{H}-6) .{ }^{13} \mathrm{C} \mathrm{NMR}\left(75 \mathrm{MHz}, \mathrm{CDCl}_{3}\right) \delta 24.9\left(2 \times \mathrm{CH}_{2}\right), 25.9$ $\left.\left(\mathrm{CH}_{2}\right), 44.2(\mathrm{C} 2), 53.8 \mathrm{COCHN}_{2}\right), 55.2(\mathrm{OMe}), 69.6\left(\mathrm{CHCl}_{2}\right), 107.3(\mathrm{C}-7), 124.2(\mathrm{C}-8 \mathrm{a}), 125.9$ (C6), 129.7 (C-4a), 133.3 (C5), 158.1 (C8), $197.0\left(\right.$ COCHN $\left._{2}\right)$.

The oil was dissolved in THF $(65 \mathrm{~mL})$, triethylamine $(6 \mathrm{~mL})$ and water $(1.5 \mathrm{~mL})$, and the resultant solution was stirred for $16 \mathrm{~h}$. Most of the THF was removed in vacuo and the residue dissolved in dichloromethane. The solution was washed with water and brine. Evaporation of the solvent gave an orange oil, which was chromatographed on silica gel, using $50 \%$ ethyl acetate/hexane as eluent, to give in the following order of elution:

(1) (2-RS)-2-Chloromethyl 5-formyl-8-methoxy-1,2,3,4-tetrahydronaphthalen-2-yl ketone as a white solid (265 mg, 12\%); a small sample was recrystallized from ether as white needles, $\mathrm{mp}$ 
145-147 ${ }^{\circ} \mathrm{C} .{ }^{1} \mathrm{H}$ NMR $\left(300 \mathrm{MHz}, \mathrm{CDCl}_{3}\right) \delta 1.75(1 \mathrm{H}, \mathrm{m}, \mathrm{H}-3), 2.20$ (1H, m, H-3), 2.70 (1H, dd, $J=12.0,18.2 \mathrm{~Hz}, \mathrm{H}-1), 2.95-3.20$ (3H, m, H-1, H-2 and H-4), 3.54 (1H, dt, $J=4.6,13.7 \mathrm{~Hz}, \mathrm{H}-$ 4), 3.92 (3H, s, OMe), $4.29\left(2 \mathrm{H}, \mathrm{d}, J=1.3 \mathrm{~Hz}, \mathrm{COCH}_{2} \mathrm{Cl}\right), 6.85(1 \mathrm{H}, \mathrm{d}, J=8.7 \mathrm{~Hz}, \mathrm{H}-7), 7.68$ $(1 \mathrm{H}, \mathrm{d}, J=8.7 \mathrm{~Hz}, \mathrm{H}-6), 10.04(1 \mathrm{H}, \mathrm{s}, \mathrm{CHO}) .{ }^{13} \mathrm{C} \mathrm{NMR}\left(75 \mathrm{MHz}, \mathrm{CDCl}_{3}\right) \delta 24.6\left(\mathrm{CH}_{2}\right), 25.2$ $\left(\mathrm{CH}_{2}\right), 26.3\left(\mathrm{CH}_{2}\right), 42.9\left(\mathrm{CH}_{2} \mathrm{Cl}\right), 47.2(\mathrm{C}-2), 55.6(\mathrm{OMe}), 106.9(\mathrm{C}-7), 124.8(\mathrm{C}-8 \mathrm{a}), 127.1(\mathrm{C}-$ 4a), 134.9 (C-6), 139.6 (C-5), 161.5 (C-8), 191.9 (CHO), $204.4\left(\mathrm{COCH}_{2} \mathrm{Cl}\right) . \mathrm{MS}$ (EI) m/z 266 $\left(\mathrm{M}^{+}, 25 \%\right), 230$ (15), 217 (27), 189 (98), 187 (100), 161 (41), 144 (31), 128 (36), 115 (72), 91 (70), 77 (50), 63 (30), 51 (46). HRMS: found 266.0710; $\mathrm{C}_{14} \mathrm{H}_{15}{ }^{35} \mathrm{ClO}_{3}$ requires 266.0709.

(2) Diazoketone 8 as yellow crystals $(1.39 \mathrm{~g}, 63 \%)$. A small sample was recrystallized from ether as yellow prisms, mp $126-128^{\circ} \mathrm{C}$. IR $\left(\mathrm{CHCl}_{3}\right) 2950,2105,1690,1640,1580,1370 \mathrm{~cm}^{-1} .{ }^{1} \mathrm{H}$ NMR $\left(300 \mathrm{MHz}, \mathrm{CDCl}_{3}\right) \delta 1.70(1 \mathrm{H}, \mathrm{m}, \mathrm{H}-3), 2.10(1 \mathrm{H}, \mathrm{m}, \mathrm{H}-3), 2.53(1 \mathrm{H}, \mathrm{m}, \mathrm{H}-2), 2.62(1 \mathrm{H}$, $\mathrm{dd}, J=10.4,17.5 \mathrm{~Hz}, \mathrm{H}-1), 2.91(1 \mathrm{H}, \mathrm{dd}, J=4.8,17.5 \mathrm{~Hz}, \mathrm{H}-1), 2.95(1 \mathrm{H}, \mathrm{dd}, J=5.5,10.8 \mathrm{~Hz}$, H-4), $3.43(1 \mathrm{H}, \mathrm{dt}, J=4.8,18.4 \mathrm{~Hz}, \mathrm{H}-4), 3.84(3 \mathrm{H}, \mathrm{s}, \mathrm{OMe}), 5.45(1 \mathrm{H}$, br s, COCHN 2$), 6.76$ $(1 \mathrm{H}, \mathrm{d}, J=8.7 \mathrm{~Hz}, \mathrm{H}-7), 7.59(1 \mathrm{H}, \mathrm{d}, J=8.7 \mathrm{~Hz}, \mathrm{H}-6), 9.96(1 \mathrm{H}, \mathrm{s}, \mathrm{CHO}) .{ }^{13} \mathrm{C} \mathrm{NMR}(75 \mathrm{MHz}$, $\left.\mathrm{CDCl}_{3}\right) \delta 25.0\left(\mathrm{CH}_{2}\right), 25.6\left(\mathrm{CH}_{2}\right), 26.1\left(\mathrm{CH}_{2}\right), 43.9(\mathrm{C} 2), 53.7\left(\mathrm{COCHN}_{2}\right), 55.4(\mathrm{OMe}), 106.7$ (C7), 125.0 (C-8a), 126.8 (C-4a), 134.3 (C6), 139.6 (C5), 161.3 (C8), 191.6 (CHO), 197.1 $\left(C_{C O C H N}\right)$. MS (EI) m/z $230\left(\mathrm{M}^{+}-\mathrm{N}_{2}, 51 \%\right), 202$ (45), 187 (55), 175 (36), 159 (35), 144 (39), 128 (52), 115 (100), 91 (87), 77 (67), 63 (56), 51 (83). Anal. Calcd for $\mathrm{C}_{14} \mathrm{H}_{14} \mathrm{~N}_{2} \mathrm{O}_{3}$ : C, 65.11; H, 5.46; N, 10.83. Found: C, 64.88; H, 5.24; N, 10.60 .

(3-RS,4a-SR,4b-RS,10b-RS)-8-Formyl-5-methoxy-2,3-dihydro-1-H-3,4b-methanocyclopropa[1,2:1,3]-dibenzene-4-(4a-H)-one (9). Diazoketone 8 (45 $\mathrm{mg}, 0.174 \mathrm{mmol})$ in dry 1,2dichloroethane $(4 \mathrm{~mL})$ was added to a solution of $\mathrm{Cu}(\mathrm{acac})_{2}(0.9 \mathrm{mg}, 2 \mathrm{~mol} \%)$ in 1,2dichloroethane $(2 \mathrm{~mL})$, heated at reflux, at a rate of $0.35 \mathrm{~mL} / \mathrm{min}$, using a syringe pump. The solution was then heated at reflux for $5 \mathrm{~min}$. To remove the copper residues, the solution was filtered through a small plug of silica gel using 25\% ethyl acetate/1,2-dichloroethane as the eluent to afford the impure norcaradiene as a yellow oil. The oil was purified using MPLC with $15 \%$ ethyl acetate/1,2-dichloroethane as eluent to give the formyl norcaradiene 9 as a yellow solid (27 mg, 65\%). A small sample was sublimed at $100{ }^{\circ} \mathrm{C}$ at $0.01 \mathrm{~mm} \mathrm{Hg}$ to give yellow needles, mp 151-153 ${ }^{\circ} \mathrm{C}$. IR $\left(\mathrm{CHCl}_{3}\right) 2950,1725,1665,1535 \mathrm{~cm}^{-1} .{ }^{1} \mathrm{H}$ NMR $\left(300 \mathrm{MHz}, \mathrm{CDCl}_{3}\right)$ $\delta 0.92(1 \mathrm{H}, \mathrm{s}, \mathrm{H}-4 \mathrm{a}), 1.80(2 \mathrm{H}, \mathrm{m}, \mathrm{H} 2 \times 2), 1.82(1 \mathrm{H}, \mathrm{d}, J=12.3 \mathrm{~Hz}, \mathrm{H}-9 \alpha), 1.98(1 \mathrm{H}, \mathrm{m}, \mathrm{H}-1 \alpha)$,

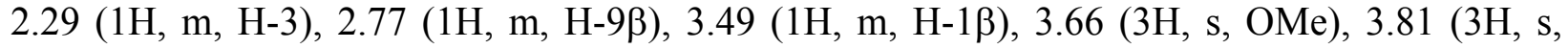
OMe), $5.36(1 \mathrm{H}, \mathrm{d}, J=7.4 \mathrm{~Hz}, \mathrm{H}-6), 6.82(1 \mathrm{H}, \mathrm{d}, J=7.4 \mathrm{~Hz}, \mathrm{H}-7), 9.38(1 \mathrm{H}$, br s, CHO $) .{ }^{13} \mathrm{C}$ NMR (75 MHz, CDCl $\left.{ }_{3}\right) \delta 18.2(\mathrm{C} 1), 27.2$ (C2), 27.7 (C9), 31.0 (C-4a), $41.2(\mathrm{C} 3), 42.4(\mathrm{C}-4 \mathrm{~b})$, 44.7 (C-8a), 56.6 (OMe), 92.6 (C6), 130.9 (C8), 145.0 (C7), 167.3 (C5), 191.0 (CHO), 214.9 (C4). MS (EI) m/z 230 (M , 99\%), 202 (71), 187(52), 174 (65), 161 (100), 146 (41), 131 (54), 115 (86), 103 (59), 91 (42), 77 (69), 63 (57), 51 (75). HRMS: found 230.0942; $\mathrm{C}_{14} \mathrm{H}_{14} \mathrm{O}_{3}$ requires 230.0943. Anal. Calcd for $\mathrm{C}_{14} \mathrm{H}_{14} \mathrm{O}_{3}$ : C, 73.03; H, 6.13. Found: C, 73.35; H, 6.18.

\section{Peterson reaction of formyl norcaradiene, 9}

Trimethylsilylmethyllithium in pentane $(1 M, 1 \mathrm{~mL})$ was added by syringe to an ice-cold solution of the cyclopropyl ketone $9(63 \mathrm{mg}, 0.274 \mathrm{mmol})$ in dry THF $(10 \mathrm{~mL})$ under a nitrogen 
atmosphere. The solution was warmed to room temperature after $2 \mathrm{~h}$. TLC analysis indicated that the starting material had been consumed and two new spots had appeared (in 10\% ethyl acetate/hexane the two spots were at $\mathrm{R}_{f} 0.5$ and 0.8 ). Potassium hydride (42 $\mathrm{mg}$ ) was added and the suspension was stirred at room temperature for $18 \mathrm{~h}$. The compound at $\mathrm{R}_{f} 0.5 \mathrm{had}$ been replaced by a spot at $\mathrm{R}_{f} 0.3$. Filtration through a very small plug of silica (to remove the potassium hydride) gave the mixture of compounds as an orange oil (60 mg). Chromatography on silica, using 10\% ethyl acetate/hexane, gave the following, in order of elution:

(1) (3-RS,4-RS,4a-SR,4b-RS,10b-RS)-4-Hydroxy-5-methoxy-4-trimethylsilylmethyl-8-vinyl2,3,4,4a-tetrahydro-1-H-3,4b-methanocyclopropa-[1,2:1,3]-dibenzene (13) (10 mg, 12\%). The compound was unstable and only the ${ }^{1} \mathrm{H}$ NMR spectrum was recorded. ${ }^{1} \mathrm{H}$ NMR $(300 \mathrm{MHz}$, $\left.\mathrm{CDCl}_{3}\right) \delta 0.08$ (9H, s, TMS), 0.52 (1H, s, H-4a), 1.00-1.45 (4H, m, $\mathrm{CH}_{2} \mathrm{TMS}$ and $\left.\mathrm{H}-2 \times 2\right), 1.12$ $(1 \mathrm{H}, \mathrm{d}, J=9.8 \mathrm{~Hz}, \mathrm{H}-9 \alpha), 1.70-2.00$ (2H, m, H-1 $\alpha$ and $\mathrm{H}-3), 2.40-2.55$ (2H, m, H-1 $\beta$ and $\mathrm{H}-$ $9 \beta), 3.62$ (3H, s, OMe), $4.90(1 \mathrm{H}, \mathrm{d}, J=6.1 \mathrm{~Hz}, \mathrm{H}-6), 4.95(1 \mathrm{H}, \mathrm{dd}, J=1.0,9.1 \mathrm{~Hz}, \mathrm{CH}=\mathrm{CH})$, $5.35\left(1 \mathrm{H}, \mathrm{dd}, J=1.0,15.2 \mathrm{~Hz}, \mathrm{CH}=\mathrm{CH}_{2}\right), 5.92(1 \mathrm{H}, \mathrm{d}, J=6.1 \mathrm{~Hz}, \mathrm{H}-7), 6.41$ (1H, dd, $J=9.1$, $15.2 \mathrm{~Hz}, \mathrm{CH}=\mathrm{CH}_{2}$ ).

(2) (3-RS,4-RS,4a-SR,4b-RS,10b-RS)-8-(Trimethylsilylethylidene)-4-hydroxy-5-methoxy-4trimethylsilylmethyl-2,3,4,4a-tetrahydro-1- $H$-3,4b-methanocyclopropa-[1,2:1,3]-dibenzen-5one (14) (9 mg, 9\%). The compound was unstable and only the ${ }^{1} \mathrm{H}$ NMR spectrum was recorded. ${ }^{1} \mathrm{H}$ NMR $\left(300 \mathrm{MHz}, \mathrm{CDCl}_{3}\right) \delta 0.02(6 \mathrm{H}, \mathrm{s}, \mathrm{TMS}), 0.08(12 \mathrm{H}, \mathrm{s}, \mathrm{TMS}), 1.05\left(2 \mathrm{H}, \mathrm{AB}\right.$ system, $\delta_{\mathrm{A}}$ $\left.=1.02, \delta_{\mathrm{B}}=1.08, J_{\mathrm{AB}}=12.0 \mathrm{~Hz}, \mathrm{CH}_{2} \mathrm{TMS}\right), 1.25\left(2 \mathrm{H}, \mathrm{s}, \mathrm{CH}_{2} \mathrm{TMS}\right), 1.40-2.10(6 \mathrm{H}, \mathrm{m}, \mathrm{H} 1 \times 2, \mathrm{H}-$ $2 \times 2, \mathrm{H}-3$ and $\mathrm{H}-4 \mathrm{a}), 2.50(2 \mathrm{H}, \mathrm{m}, \mathrm{H}-9 \beta$ and $\mathrm{H}-1 \beta), 5.78(1 \mathrm{H}, \mathrm{d}, J=1.0,9.7 \mathrm{~Hz}, \mathrm{H}-7), 6.05(1 \mathrm{H}$, $\mathrm{dt}, J=1.0,7.6 \mathrm{~Hz}, \mathrm{H}-10), 7.00(1 \mathrm{H}, \mathrm{d}, J=9.7 \mathrm{~Hz}, \mathrm{H}-6)$.

(2-RS)-E- and Z-5-Cyanovinyl-8-methoxy-1,2,3,4-tetrahydro-2-naphthoic acid (15). Diethyl cyanomethyl phosphonate $(3.2 \mathrm{~mL}, 4$ equiv.) in dry THF $(15 \mathrm{~mL})$ was added dropwise to sodium hydride $(765 \mathrm{mg})$ under a nitrogen atmosphere at $0{ }^{\circ} \mathrm{C}$. The suspension was stirred for $5 \mathrm{~min}$ and the aldehyde $7(1.5 \mathrm{~g}, 6.4 \mathrm{mmol})$ in THF $(40 \mathrm{~mL})$ was added rapidly from a dropping funnel. After stirring at $0{ }^{\circ} \mathrm{C}$ for $1 \mathrm{~h}$ the solution was warmed to room temperature and stirred for $2 \mathrm{~h}$. The reaction was quenched with water and acidified to $\mathrm{pH} 1$ with concentrated hydrochloric acid. The aqueous solution was extracted with ethyl acetate and the combined organic extracts washed with water and brine. The solvent was removed in vacuo to give a brown solid, which was purified by column chromatography using $1 \% \mathrm{CH}_{2} \mathrm{Cl}_{2} / 50 \%$ ethyl acetate $/ 49 \%$ hexane as eluent to give the mixture of acids $\mathbf{1 5}$ as a cream solid (1.48 g, 90\%). ${ }^{1} \mathrm{H}$ NMR spectroscopy revealed that there was a 3:1 mixture of $E$ - to $Z$ - isomers. Two recrystallizations of the mixture with methanol gave the E- acid as pale yellow prisms (1.02 g, 62\%), mp $189-191{ }^{\circ} \mathrm{C}$. IR $\left(\mathrm{CHCl}_{3}\right) 3000,2225,1750,1710,1590 \mathrm{~cm}^{-1}$. ${ }^{1} \mathrm{H}$ NMR $\left(300 \mathrm{MHz}, \mathrm{d}_{6}\right.$-acetone) $\delta 1.85(1 \mathrm{H}, \mathrm{m}, \mathrm{H}-$ 3), $2.28(1 \mathrm{H}, \mathrm{m}, \mathrm{H}-3), 2.63-2.85(3 \mathrm{H}, \mathrm{m}, \mathrm{H}-2, \mathrm{H}-1$ and $\mathrm{H}-4), 2.96(1 \mathrm{H}, \mathrm{dt}, J=5.0,17.6 \mathrm{~Hz}, \mathrm{H}-4)$, $3.08(1 \mathrm{H}, \mathrm{m}, \mathrm{H}-1), 3.84(3 \mathrm{H}, \mathrm{s}, \mathrm{OMe}), 5.66(1 \mathrm{H}, \mathrm{d}, J=16.4 \mathrm{~Hz}, \mathrm{CH}=\mathrm{CHCN}), 6.71(1 \mathrm{H}, \mathrm{d}, J=$ $8.6 \mathrm{~Hz}, \mathrm{H}-7), 7.36(1 \mathrm{H}, \mathrm{d}, J=8.6 \mathrm{~Hz}, \mathrm{H}-6), 7.62(1 \mathrm{H}, \mathrm{d}, J=16.4 \mathrm{~Hz}, \mathrm{CH}=\mathrm{CHCN}) .{ }^{13} \mathrm{C} \mathrm{NMR}(75$ $\mathrm{MHz}, \mathrm{d}_{6}$-acetone) $\delta 24.8\left(\mathrm{CH}_{2}\right), 25.6\left(\mathrm{CH}_{2}\right), 25.8\left(\mathrm{CH}_{2}\right), 38.3(\mathrm{C} 2), 55.4(\mathrm{OMe}), 94.5$ $(\mathrm{CH}=\mathbf{C H C N}), 107.5(\mathrm{C} 7), 118.8(\mathrm{CN}), 124.6(\mathrm{C}-8 \mathrm{a}$ or $\mathrm{C}-4 \mathrm{a}), 124.7(\mathrm{C} 6), 124.8(\mathrm{C}-4 \mathrm{a}$ or $\mathrm{C}-8 \mathrm{a})$, 
$136.0(\mathrm{C} 5), 147.8(\mathrm{CH}=\mathrm{CHCN}), 159.5(\mathrm{C} 8), 179.1\left(\mathrm{CO}_{2} \mathrm{H}\right) . \mathrm{MS}(\mathrm{EI}) \mathrm{m} / \mathrm{z} 257\left(\mathrm{M}^{+}, 32 \%\right), 210$ (59), 195 (59), 180 (100), 167 (22), 153 (23), 140 (42), 128 (40), 115 (91), 102 (18), 84 (71), 77 (44), 63 (60), 51 (65). Anal. Calcd for $\mathrm{C}_{15} \mathrm{H}_{15} \mathrm{NO}_{3}$ : C, 70.02; H, 5.88; N, 5.44. Found: C, 70.17; $\mathrm{H}, 6.16 ; \mathrm{N}, 5.12$.

Concentration of the mother liquor gave the Z-acid as white prisms $(0.43 \mathrm{~g}, 26 \%)$, mp 196$198 \mathrm{C}$. IR $\left(\mathrm{CHCl}_{3}\right)$ 3000, 2225, 1750, 1710, $1590 \mathrm{~cm}^{-1} .{ }^{1} \mathrm{H}$ NMR (300 MHz, $\mathrm{d}_{6}$-acetone) $\delta 1.82$ (1H, m, H-3), 2.28 (1H, m, H-3), 2.66-2.85 (3H, m, H-2, H-1 and H-4), $2.86(1 \mathrm{H}, \mathrm{dt}, J=5.0$, $17.6 \mathrm{~Hz}, \mathrm{H}-4), 3.04(1 \mathrm{H}, \mathrm{m}, \mathrm{H}-1), 3.85(3 \mathrm{H}, \mathrm{s}, \mathrm{OMe}), 5.48(1 \mathrm{H}, \mathrm{d}, J=12.0 \mathrm{~Hz}, \mathrm{CH}=\mathrm{CHCN})$, $6.91(1 \mathrm{H}, \mathrm{d}, J=8.7 \mathrm{~Hz}, \mathrm{H}-7), 7.40(1 \mathrm{H}, \mathrm{d}, J=8.7 \mathrm{~Hz}, \mathrm{H}-6), 7.76(1 \mathrm{H}, \mathrm{d}, J=12.0 \mathrm{~Hz}$, $\mathrm{CH}=\mathrm{CHCN}) .{ }^{13} \mathrm{C} \mathrm{NMR}\left(75 \mathrm{MHz}, \mathrm{d}_{6}\right.$-acetone) $\delta 26.3\left(\mathrm{CH}_{2}\right), 27.1\left(\mathrm{CH}_{2}\right), 27.3\left(\mathrm{CH}_{2}\right), 39.6(\mathrm{C}-2)$, $56.5(\mathrm{OMe}), 95.9(\mathrm{CH}=\mathrm{CHCN}), 108.4(\mathrm{C} 7), 118.9(\mathrm{CN}), 125.9(\mathrm{C}-8 \mathrm{a}$ or $\mathrm{C}-4 \mathrm{a}), 126.5(\mathrm{C}-4 \mathrm{a}$ or C-8a), 127.9 (C6), 137.7 (C5), $148.2(\mathrm{CH}=\mathrm{CHCN}), 160.3(\mathrm{C} 8), 177.4\left(\mathrm{CO}_{2} \mathrm{H}\right) . \mathrm{MS}(\mathrm{EI}) \mathrm{m} / \mathrm{z} 257$ $\left(\mathrm{M}^{+}, 80 \%\right), 226$ (17), 210 (94), 195 (69), 180 (100), 153 (28), 140 (30), 127 (27), 115 (63), 77 (37), 63 (41), 51 (43). HRMS: found 257.1053; $\mathrm{C}_{15} \mathrm{H}_{15} \mathrm{NO}_{3}$ requires 257.1052.

(2-RS)-E-Diazomethyl-5-cyanovinyl-8-methoxy-1,2,3,4-tetrahydronaphthalen-2-yl ketone (16). A solution of the E-acid 15 (1.02 g, $3.97 \mathrm{mmol})$ in dichloromethane $(20 \mathrm{~mL})$ was added dropwise to a solution of oxalyl chloride $(5 \mathrm{~mL})$ and dichloromethane $(5 \mathrm{~mL})$ at room temperature under a nitrogen atmosphere. One drop of dry DMF was added (evolution of gas) and the resultant solution stirred for $14 \mathrm{~h}$. The volatile components were removed in vacuo and the procedure repeated twice more to afford the acid chloride as an orange solid. After $1 \mathrm{~h}$ under high vacuum, to remove any traces of $\mathrm{HCl}$, the oil was dissolved in dichloromethane $(30 \mathrm{~mL})$ and added to fresh ethereal diazomethane at $-20 \mathrm{C}$ (methanol/ice bath) under nitrogen. After stirring for $15 \mathrm{~h}$ the yellow solution was filtered through Celite in a well-ventilated fume-hood. Evaporation of the solvent in vacuo gave an orange oil, which was chromatographed on silica gel using 50\% ethyl acetate/5\% 1,2-dichloroethane/45\% hexane as eluent to give the diazoketone 16 as yellow crystals $(796 \mathrm{mg}, 71 \%)$. A small sample was recrystallized from ether to give yellow needles, mp 139-141 C. IR $\left(\mathrm{CHCl}_{3}\right)$ 2950, 2225, 2120, 1645, $1590 \mathrm{~cm}^{-1}$. ${ }^{1} \mathrm{H}$ NMR (300 MHz, $\left.\mathrm{CDCl}_{3}\right) \delta 1.82(1 \mathrm{H}, \mathrm{m}, \mathrm{H}-3), 2.15(1 \mathrm{H}, \mathrm{m}, \mathrm{H}-3), 2.55$ (1H, m, H-2), 2.60-2.80 (2H, m, H-4×2), $2.93(2 \mathrm{H}, \mathrm{m}, \mathrm{H}-1 \times 2), 3.84(3 \mathrm{H}, \mathrm{s}, \mathrm{OMe}), 5.42\left(1 \mathrm{H}\right.$, br s, $\left.\mathrm{COCHN}_{2}\right), 5.66(1 \mathrm{H}, \mathrm{d}, J=16.4 \mathrm{~Hz}$, $\mathrm{CH}=\mathrm{CHCN}), 6.71(1 \mathrm{H}, \mathrm{d}, J=8.8 \mathrm{~Hz}, \mathrm{H}-7), 7.35(1 \mathrm{H}, \mathrm{d}, J=8.8 \mathrm{~Hz}, \mathrm{H}-6), 7.60(1 \mathrm{H}, \mathrm{d}, J=16.4$ $\mathrm{Hz}, \mathrm{CH}=\mathrm{CHCN}) .{ }^{13} \mathrm{C} \mathrm{NMR}\left(75 \mathrm{MHz}, \mathrm{CDCl}_{3}\right) \delta 25.1\left(\mathrm{CH}_{2}\right), 25.9\left(\mathrm{CH}_{2}\right), 26.0\left(\mathrm{CH}_{2}\right), 44.2(\mathrm{C} 2)$, $53.9\left(\mathrm{COCHN}_{2}\right), 55.4(\mathrm{OMe}), 94.5(\mathrm{CH}=\mathrm{CHCN}), 107.5(\mathrm{C}-7), 118.8(\mathrm{CN}), 124.7(\mathrm{C}-6), 124.7$ $\left(\mathrm{C}-8 \mathrm{a}\right.$ and C-4a), $136.0(\mathrm{C}-5), 147.7(\mathrm{CH}=\mathrm{CHCN}), 159.4(\mathrm{C}-8), 196.9\left(\mathrm{COCHN}_{2}\right) . \mathrm{MS}(\mathrm{EI}) \mathrm{m} / \mathrm{z}$ $281\left(\mathrm{M}^{+}, 2 \%\right), 253$ (45), 210 (85), 195 (42), 155 (75), 140 (67), 128 (51), 115 (100), 77 (48), 63 (66), 51 (73). HRMS: found 281.1165; $\mathrm{C}_{16} \mathrm{H}_{15} \mathrm{~N}_{3} \mathrm{O}_{2}$ requires 281.1164. Anal. Calcd for $\mathrm{C}_{16} \mathrm{H}_{15} \mathrm{~N}_{3} \mathrm{O}_{2}$ : C, 68.31; H, 5.37; N, 14.94. Found: C, 67.97; H, 5.42; N, 14.71.

\section{(3-RS,4a-SR,4b-RS,10b-RS)-E-8-Cyanovinyl-5-methoxy-2,3,4,4a-tetrahydro-1H-3,4b-}

methanocyclopropa-[1,2:1,3]-dibenzene-4-one (17). The diazoketone 16 (796 mg, $2.83 \mathrm{mmol}$ ) in dry 1,2-dichloroethane $(20 \mathrm{~mL})$ was added to a solution of $\mathrm{Cu}(\mathrm{acac})_{2}(7 \mathrm{mg}, 2 \mathrm{~mol} \%)$ in 1,2dichloroethane $(20 \mathrm{~mL})$, at reflux, at a rate of $0.15 \mathrm{~mL} / \mathrm{min}$ using a syringe pump. Upon addition 
the solution was refluxed for $5 \mathrm{~min}$. To remove the copper residues the solution was filtered through a small plug of silica gel using 50\% ethyl acetate/1,2-dichloroethane as eluent to afford the impure norcaradiene as a yellow oil. The oil was purified using MPLC with 30\% ethyl acetate/65\% hexane/5\% 1,2-dichloroethane as eluent to give the cyano-norcaradiene 17 as a yellow solid (430 mg, 60\%). A small sample was recrystallized from ether as pale yellow needles, mp 130-132 ${ }^{\circ} \mathrm{C}$. IR $\left(\mathrm{CHCl}_{3}\right) 2950,2220,1720,1540 \mathrm{~cm}^{-1} .{ }^{1} \mathrm{H} \mathrm{NMR}\left(300 \mathrm{MHz}, \mathrm{CDCl}_{3}\right)$ $\delta 0.95(1 \mathrm{H}, \mathrm{d}, J=1.6 \mathrm{~Hz}, \mathrm{H}-4 \mathrm{a}), 1.81(1 \mathrm{H}, \mathrm{d}, J=12.4 \mathrm{~Hz}, \mathrm{H}-9 \alpha), 1.80-2.05(3 \mathrm{H}, \mathrm{m}, \mathrm{H}-2 \times 2, \mathrm{H}-$

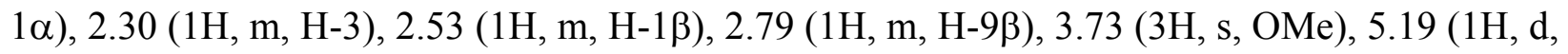
$J=7.2 \mathrm{~Hz}, \mathrm{H}-6), 5.44(1 \mathrm{H}, \mathrm{d}, J=16.4 \mathrm{~Hz}, \mathrm{CH}=\mathrm{CHCN}), 6.35(1 \mathrm{H}, \mathrm{d}, J=7.2 \mathrm{~Hz}, \mathrm{H}-7), 7.09(1 \mathrm{H}$, $\mathrm{d}, J=16.4 \mathrm{~Hz}, \mathrm{CH}=\mathrm{CHCN}) .{ }^{13} \mathrm{C} \mathrm{NMR}\left(75 \mathrm{MHz}, \mathrm{CDCl}_{3}\right) \delta 19.3(\mathrm{C} 1), 27.2$ (C-2), 27.5 (C-9), 29.6 (C-4a), 40.7 (C-3), 42.0 (C-4b or -8a), 44.2 (C-8a or -4b), $56.1(\mathrm{OMe}), 92.6$ (C-6), 93.2 $(\mathrm{CH}=\mathbf{C H C N}), 118.8(\mathrm{CN}), 127.0(\mathrm{C}-8), 128.4(\mathrm{C}-7), 148.2(\mathrm{CH}=\mathrm{CHCN}), 162.6(\mathrm{C}-5), 214.9(\mathrm{C}-$ 4). MS (EI) m/z 253 (M $\left.\mathrm{M}^{+}, 100 \%\right), 202$ (71), 187(52), 174 (65), 161 (100), 146 (41), 131 (54), 115 (86), 103 (59), 91 (42), 77 (69), 63 (57), 51 (75). Anal. Calcd for $\mathrm{C}_{16} \mathrm{H}_{15} \mathrm{NO}_{2}$ : C, 75.87; H, 5.97; N, 5.53. Found: C, 75.61; H, 5.96; N, 5.72.

(3-RS,4a-SR,4b-RS,6a-SR,7-RS,8-SR,9-SR,10b-RS)-9-Cyano-5-methoxy-2,3,6a,7,8,9hexahydro-1-H-3,4b-methanobenzo-[1,3]-cyclopropa-[1,2-a]-naphthalen-4(4a-H)-one-7,8dicarboxylic anhydride (18). The norcaradiene 17 (300 $\mathrm{mg}, 1.19 \mathrm{mmol})$ and freshly sublimed maleic anhydride (250 mg, 2.6 equiv.) were dissolved in dry benzene $(30 \mathrm{~mL})$. The solution was heated at $80^{\circ} \mathrm{C}$ for $48 \mathrm{~h}$, in which time a white solid precipitated from the reaction mixture. TLC analysis indicated that all of the starting material had been consumed. The solvent was removed on the rotary evaporator to afford a yellow solid (450 mg, >100\%). ${ }^{1} \mathrm{H}$ NMR spectroscopy revealed that only one diastereoisomer had been formed. The solid was recrystallized from acetone to afford the adduct 18 as fine white needles (365 mg, 88\%), m.p. $168-170{ }^{\circ} \mathrm{C} .{ }^{1} \mathrm{H}$ NMR

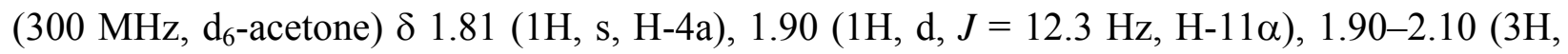
m, H-2 ×2 and H-1 $\alpha), 2.25$ (1H, m, H-3), $2.69(1 \mathrm{H}, \mathrm{m}, \mathrm{H}-1 \beta), 2.78(1 \mathrm{H}, \mathrm{m}, \mathrm{H}-11 \beta), 3.35(1 \mathrm{H}, \mathrm{m}$, H-6a), 3.78 (3H, s, OMe), 3.94 (1H, dd, $J=6.8,9.3 \mathrm{~Hz}, \mathrm{H}-7), 4.05$ (1H, m, H-9), 4.20 (1H, dd, $J$ $=5.3,9.3 \mathrm{~Hz}, \mathrm{H}-8), 4.89(1 \mathrm{H}, \mathrm{d}, J=3.3 \mathrm{~Hz}, \mathrm{H}-6), 6.39(1 \mathrm{H}, \mathrm{m}, \mathrm{H}-10) .{ }^{13} \mathrm{C} \mathrm{NMR}\left(75 \mathrm{MHz}, \mathrm{d}_{6^{-}}\right.$ acetone) $\delta 18.0(\mathrm{C} 1), 25.1(\mathrm{C}-4 \mathrm{a}), 25.8$ (C2), 26.1 (C-9), 33.1 (C-6a), 35.0 (C-4b or C-10b), 37.9 (C-10b or C-4b), 39.6 (C-7), 42.1 (C-8), 42.8 (C-3), 44.7 (C-9), 53.3 (OMe), 88.2 (C-6), 117.0 (CN), 119.7 (C-10), 139.4 (C-10a), 151.3 (C-5), 168.3 (C-12 or C-13), 169.6 (C-13 or C-12) 208.5 (C-4). MS (EI) m/z 351 (M+, 28\%), 323 (10), 278 (17), 253 (88), 236 (34), 222 (25), 210 (28), 197 (30), 166 (43), 153 (30), 140 (40), 127 (30), 115 (34), 89 (31), 77 (49), 63 (47), 55 (100), 51 (56). HRMS: found 351.11111; $\mathrm{C}_{20} \mathrm{H}_{17} \mathrm{NO}_{5}$ requires 351.1108.

Methyl 5-hydroxy-1,2,3,4-tetrahydro-2-naphthoate. To a solution of 1,2,3,4-tetrahydro-5hydroxy-2-naphthoic acid $19^{16}(4.0 \mathrm{~g}, 21 \mathrm{mmol})$ in ether $(50 \mathrm{~mL})$ was added an ethereal solution of diazomethane, until a persistent yellow coloration was observed. A drop of acetic acid was added and the excess diazomethane removed by bubbling nitrogen gas through the solution. The reaction mixture was filtered through a pad of Celite followed by removal of the solvent in vacuo. Purification by column chromatography (20\% ethyl acetate/petrol) afforded methyl 5- 
hydroxy-1,2,3,4-tetrahydro-2-naphthoate (3.95 g, 92\%) as an off-white solid. IR (soln.) 3400$3200(\mathrm{O}-\mathrm{H}), 3160(\mathrm{ArC}-\mathrm{H}), 2960(\mathrm{C}-\mathrm{H}), 1725(\mathrm{C}=\mathrm{O}), 1590(\mathrm{ArC}=\mathrm{C}) \mathrm{cm}^{-1} .{ }^{1} \mathrm{H}$ NMR $\delta 7.00$ $(1 \mathrm{H}, \mathrm{t}, J=7.9 \mathrm{~Hz}, \mathrm{H}-7), 6.71(1 \mathrm{H}, \mathrm{d}, J=7.9 \mathrm{~Hz}, \mathrm{H}-8), 6.61(1 \mathrm{H}, \mathrm{d}, J=7.9 \mathrm{~Hz}, \mathrm{H}-6), 5.01(1 \mathrm{H}$, br s, OH), $3.73\left(1 \mathrm{H}, \mathrm{s}, \mathrm{OCH}_{3}\right), 3.00-2.97(2 \mathrm{H}, \mathrm{m}, \mathrm{H}-1), 2.88-2.86(1 \mathrm{H}, \mathrm{m}, \mathrm{H}-4), 2.72-2.60(2 \mathrm{H}$, $\mathrm{m}, \mathrm{H}-4$ and $\mathrm{H}-2), 2.32-2.23(1 \mathrm{H}, \mathrm{m}, \mathrm{H}-3), 1.91-1.77(1 \mathrm{H}, \mathrm{m}, \mathrm{H}-3) .{ }^{13} \mathrm{C} \mathrm{NMR} \delta 176.2(\mathrm{C}=\mathrm{O})$, 153.3 (C5), 136.6 (C-8a), 126.4 (C7), 122.4 (C-4a), 121.3 (C8), 121.1 (C6), $51.9\left(\mathrm{OCH}_{3}\right), 39.6$ (C2), 31.7 and 25.4 (C1 and C4), 22.3 (C3). LRMS $206\left(\mathrm{M}^{+}, 46 \%\right), 191\left(\mathrm{M}-\mathrm{CH}_{3}, 2\right), 175(\mathrm{M}-$ $\left.\mathrm{OCH}_{3}, 7\right), 146$ (M-MeOH, CO, 100), 131 (32), 115 (22), 107 (18), 91 (26). HRMS: found (M ) 206.0943; $\mathrm{C}_{12} \mathrm{H}_{14} \mathrm{O}_{3}$ requires 206.0943.

Methyl 5-(trifluoromethanesulfonyloxy)-1,2,3,4-tetrahydro-2-naphthoate (20). To a solution of the phenol $(3.65 \mathrm{~g}, 18 \mathrm{mmol})$ in pyridine $(25 \mathrm{~mL})$ at $0 \mathrm{C}$ was added dropwise trifluoromethanesulfonic anhydride (freshly made from the acid: $4.5 \mathrm{~mL}, 1.5$ equiv.) with fuming! The resulting red solution was allowed to warm overnight to room temperature, with stirring. The reaction mixture was poured into water and the aqueous layer extracted with ether $(\times 3)$. The combined ethereal extracts were washed successively with water $(\times 2), \mathrm{HCl}(1 \mathrm{M}$, aq.), water, $\mathrm{NaCl}$ (satd. aq.) and finally dried over $\mathrm{MgSO}_{4}$. Removal of the solvent under reduced pressure, followed by purification using column chromatography (5\% ethyl acetate/petrol) afforded methyl 5-(trifluoromethanesulfonyloxy)-1,2,3,4-tetrahydro-2-naphthoate (20) (5.0 g, $83 \%)$ as a colorless oil, which formed white crystals when stored at $4 \mathrm{C}$. IR (soln.) $3030(\mathrm{C}=\mathrm{C}-$ $\mathrm{H}), 2960(\mathrm{C}-\mathrm{H}), 1760(\mathrm{C}=\mathrm{O}), 1610$ and $1550(\mathrm{ArC}=\mathrm{C}), 1410$ and $1210(\mathrm{~S}=\mathrm{O}), 1280(\mathrm{C}-\mathrm{F}) \mathrm{cm}^{-1}$. ${ }^{1} \mathrm{H}$ NMR $\delta 7.15(3 \mathrm{H}, \mathrm{m}, \mathrm{Ar}-\mathrm{H}), 3.74\left(3 \mathrm{H}, \mathrm{s}, \mathrm{OCH}_{3}\right), 3.11-3.04(2 \mathrm{H}, \mathrm{m}, \mathrm{H}-1), 3.06-2.97$ (1H, m, $\mathrm{H}-4), 2.82-2.70$ (2H, m, H-4 and H-2), 2.33-2.23 (1H, m, H-3), 1.91-1.79 (1H, m, H-3). ${ }^{13} \mathrm{C}$ NMR $\delta 175.0(\mathrm{C}=\mathrm{O}), 148.0$ (C5), 138.4 (C-8a), 129.0 (C8), 128.9 (C-4a), 126.9 (C7), 118.6 (C6), $118.5\left(\mathrm{q}, J_{\mathrm{C}-\mathrm{F}}=320 \mathrm{~Hz}, \mathrm{CF}_{3}\right), 51.9\left(\mathrm{OCH}_{3}\right), 38.9(\mathrm{C} 2), 31.3$ and $24.8(\mathrm{C} 1$ and $\mathrm{C} 4), 22.8$ (C3). LRMS $338\left(\mathrm{M}^{+}, 24 \%\right), 306(\mathrm{M}-\mathrm{MeOH}, 20), 278$ (M-MeOH, CO, 45), $145(\mathrm{M}-\mathrm{MeOH}$, $\left.\mathrm{CO}, \mathrm{SO}_{2} \mathrm{CF}_{3}, 100\right), 69\left(\mathrm{CF}_{3}, 15\right)$. HRMS: found $\left(\mathrm{M}^{+}\right)$338.0437; $\mathrm{C}_{13} \mathrm{H}_{13} \mathrm{~F}_{3} \mathrm{O}_{5} \mathrm{~S}$ requires 338.0436.

Methyl 5-vinyl-1,2,3,4-tetrahydro-2-naphthoate (21). To a solution of the triflate $(5.0 \mathrm{~g}$, $15 \mathrm{mmol})$, lithium chloride (1.9 g, $45 \mathrm{mmol}, 3$ equiv. $)$ and palladium tetrakis(triphenylphosphine) (870 mg, $0.75 \mathrm{mmol}, 5 \mathrm{~mol} \%)$ in dimethylformamide $(50 \mathrm{~mL})$ was added vinyl tri-n-butylstannane $(5.3 \mathrm{~mL}, 18 \mathrm{mmol}, 1.2$ equiv.). The resulting pale yellow solution was heated to $100 \mathrm{C}$ for 24 hours, during which the consumed catalyst was observed to form a black precipitate. After cooling to room temperature the reaction mixture was poured into $\mathrm{NH}_{4} \mathrm{OH}$ solution $(25 \%$, aq) and the aqueous layer extracted with ether $(\times 3)$. The combined ethereal extracts were washed successively with additional $\mathrm{NH}_{4} \mathrm{OH}(25 \%, a q)$, water $(\times 2), \mathrm{HCl}(1 \mathrm{M}$, aq.), water, $\mathrm{NaCl}$ (satd aq.) and finally dried over $\mathrm{MgSO}_{4}$. Removal of the solvent under reduced pressure, followed by purification using column chromatography (5\% ethyl acetate/petrol) afforded the vinyl ester $21(2.7 \mathrm{~g}, 84 \%)$ as a colorless oil, IR (soln) 3090 and $3060(\mathrm{C}=\mathrm{C}-\mathrm{H})$, $2930(\mathrm{C}-\mathrm{H}), 1730(\mathrm{C}=\mathrm{O}), 1630(\mathrm{C}=\mathrm{C}), 1580(\mathrm{ArC}=\mathrm{C}) \mathrm{cm}^{-1} .{ }^{1} \mathrm{H}$ NMR $\delta 7.32(1 \mathrm{H}, \mathrm{d}, J=7.6 \mathrm{~Hz}$, H-6), $7.13(1 \mathrm{H}, \mathrm{t}, J=7.6 \mathrm{~Hz}, \mathrm{H}-7), 7.05(1 \mathrm{H}, \mathrm{d}, J=7.6 \mathrm{~Hz}, \mathrm{H}-8), 6.91(1 \mathrm{H}, \mathrm{dd}, J=17.3,10.9$ Hz, H-5a), 5.61 (1H, d, $J=17.3 \mathrm{~Hz}, \mathrm{H}-5 \mathrm{~b}-$ trans), 5.30 (1H, d, $J=10.9 \mathrm{~Hz}, \mathrm{H}-5 \mathrm{~b}-$ cis), 3.74 (3H, 
s, $\left.\mathrm{OCH}_{3}\right), 3.03(2 \mathrm{H}, \mathrm{d}, J=8.1 \mathrm{~Hz}, \mathrm{H}-1), 3.01-2.91(1 \mathrm{H}, \mathrm{m}, \mathrm{H}-4), 2.80-2.66(2 \mathrm{H}, \mathrm{m}, \mathrm{H}-4$ and H2), 2.31-2.25 (1H, m, H-3), 1.92-1.80 (1H, m, H-3). ${ }^{13} \mathrm{C}$ NMR $\delta 175.8(\mathrm{C}=\mathrm{O}), 137.0$ (C5), 135.0 (C-8a), 134.6 (C-5a), 132.9 (C-4a), 128.7 (C6), 125.8 (C8), 123.6 (C7), 115.8 (C-5b), 51.8 $\left(\mathrm{OCH}_{3}\right), 39.4(\mathrm{C} 2), 32.2,25.9$ and $25.8\left(3 \times \mathrm{CH}_{2}\right)$. LRMS $216\left(\mathrm{M}^{+}, 70 \%\right), 185\left(\mathrm{M}-\mathrm{OCH}_{3}, 10\right)$, 156 (M-MeOH, CO, 100), 141 (73), 129 (54), 115 (50). HRMS: found ( $\mathrm{M}^{+}$) 216.1150; $\mathrm{C}_{14} \mathrm{H}_{16} \mathrm{O}_{2}$ requires 216.1150 .

5-Vinyl-1,2,3,4-tetrahydro-2-naphthoic acid. To a solution of the methyl ester (2.6 g, $12 \mathrm{mmol})$ in methanol $(50 \mathrm{~mL})$ was added sodium hydroxide solution $(4 \mathrm{M}$, aq, from $14 \mathrm{~g} \mathrm{NaOH}$ in $100 \mathrm{~mL}, 30$ equiv.). After 5 hours' stirring at room temperature the reaction mixture was acidified to $\mathrm{pH} 1$. The aqueous solution was extracted with ethyl acetate $(\times 3)$ and the combined organic layers washed with $\mathrm{NaCl}$ (satd aq.) and dried over $\mathrm{MgSO}_{4}$. Removal of the solvent under reduced pressure afforded 5-vinyl-1,2,3,4-tetrahydro-2-naphthoic acid (2.25 g, 94\%) as a white solid. A small sample was recrystallized from ethyl acetate to afford white crystals, mp 118120 C. IR (soln) 3400-3100 (O-H), 3090 and $3060(\mathrm{C}=\mathrm{C}-\mathrm{H}), 2940(\mathrm{C}-\mathrm{H}), 1710(\mathrm{C}=\mathrm{O}), 1625$ $(\mathrm{C}=\mathrm{C}), 1580(\mathrm{ArC}=\mathrm{C}) \mathrm{cm}^{-1} .{ }^{1} \mathrm{H}$ NMR $\delta 7.35(1 \mathrm{H}, \mathrm{d}, J=7.4 \mathrm{~Hz}, \mathrm{H}-6), 7.15(1 \mathrm{H}, \mathrm{t}, J=7.4 \mathrm{~Hz}$, H-7), $7.07(1 \mathrm{H}, \mathrm{d}, J=7.4 \mathrm{~Hz}, \mathrm{H}-8), 6.93(1 \mathrm{H}, \mathrm{dd}, J=17.4,11.0 \mathrm{~Hz}, \mathrm{H}-5 \mathrm{a}), 5.63(1 \mathrm{H}, \mathrm{dd}, J=$ 17.4, $1.5 \mathrm{~Hz}, \mathrm{H}-5 \mathrm{~b}$-trans), $5.32(1 \mathrm{H}, \mathrm{dd}, J=11.0,1.5 \mathrm{~Hz}, \mathrm{H}-5 \mathrm{~b}-$ cis $), 3.07$ (2H, d, $J=8.2 \mathrm{~Hz}, \mathrm{H}-$ 1), 3.05-2.98 (1H, m, H-4), 2.82-2.72 (2H, m, H-4 and H-2), 2.38-2.28 (1H, m, H-3), 1.99-1.84 (1H, m, H-3). ${ }^{13} \mathrm{C}$ NMR $\delta 181.9(\mathrm{C}=\mathrm{O}), 137.1$ (C5), 134.7 (C-8a), 134.5 (C-5a), 132.8 (C-4a), 128.8 (C8), 125.9 (C6), 123.7 (C7), 115.9 (C-5b), $39.3(\mathrm{C} 2), 31.9,25.8$ and $25.7\left(3 \times \mathrm{CH}_{2}\right)$. LRMS $202\left(\mathrm{M}^{+}, 100 \%\right), 187\left(\mathrm{M}-\mathrm{CH}_{3}, 8\right), 176(4), 156\left(\mathrm{M}-\mathrm{H}_{2} \mathrm{O}, \mathrm{CO}, 77\right), 141\left(\mathrm{M}-\mathrm{H}_{2} \mathrm{O}, \mathrm{CH}_{3}\right.$, $\mathrm{CO}, 73), 129$ (93), 115 (63). HRMS: found $\left(\mathrm{M}^{+}\right)$202.0994; $\mathrm{C}_{13} \mathrm{H}_{14} \mathrm{O}_{2}$ requires 202.0994.

5-Vinyl-1,2,3,4-tetrahydro-2-naphthoyl chloride. To a solution of the acid (2.1 g, $11 \mathrm{mmol})$ in dichloromethane $(50 \mathrm{~mL})$ was added oxalyl chloride $(10 \mathrm{~mL}, 120 \mathrm{mmol}, 10$ equiv. $)$ and a single drop of dimethylformamide. After stirring at room temperature for 18 hours, the solvent was removed under reduced pressure, and the residue azeotroped with benzene $(\times 3)$ [to remove residual oxalyl chloride and $\mathrm{HCl}]$. The residue was dried under high vacuum for 1 hour, and the resulting orange oil was used directly in the next step. ${ }^{1} \mathrm{H}$ NMR $\delta 7.35(1 \mathrm{H}, \mathrm{d}, J=7.4 \mathrm{~Hz}, \mathrm{H}-6)$, $7.15(1 \mathrm{H}, \mathrm{t}, J=7.4 \mathrm{~Hz}, \mathrm{H}-7), 7.07$ (1H, d, $J=7.4 \mathrm{~Hz}, \mathrm{H}-8), 6.93(1 \mathrm{H}, \mathrm{dd}, J=17.4,11.0 \mathrm{~Hz}, \mathrm{H}-$ 5a), 5.63 (1H, dd, $J=17.4,1.5 \mathrm{~Hz}, \mathrm{H}-5 \mathrm{~b}$-trans), 5.32 (1H, dd, $J=11.0,1.5 \mathrm{~Hz}, \mathrm{H}-5 \mathrm{~b}-$-cis), 3.20$3.08(2 \mathrm{H}, \mathrm{m}, \mathrm{H}-1), 3.04-2.95$ (1H, m, H-4), 2.83-2.73 (2H, m, H-4 and H-2), 2.48-2.39 (1H, m, H-3), 2.07-1.96 (1H, m, H-3). ${ }^{13} \mathrm{C}$ NMR $\delta 176.4(\mathrm{C}=\mathrm{O}), 137.1$ (C5), 134.2 (C-5a), 133.4 and 132.2 (C-8a and C-4a), 128.8 (C-8), 126.1 (C-6), 123.9 (C-7), 116.1 (C-5b), 51.3 (C-2), 32.2, 25.9 and $25.3\left(3 \times \mathrm{CH}_{2}\right)$.

Diazomethyl 5-vinyl-1,2,3,4-tetrahydronaphthalen-2-yl ketone. To an ethereal solution of diazomethane at $-20 \mathrm{C}$ was added dropwise a solution of the crude acid chloride in dichloromethane $(50 \mathrm{~mL})$. The solution was allowed to warm gradually to room temperature with further stirring over 5 hours. Excess diazomethane was removed by bubbling nitrogen gas through the solution, after which the solution was filtered through a pad of Celite and the solvent removed in vacuo. Purification by column chromatography $(20 \%$ ethyl acetate/petrol) afforded 
diazomethyl 5-vinyl-1,2,3,4-tetrahydronaphthalen-2-yl ketone $(2.0 \mathrm{~g}, 80 \%$ from the acid) as a yellow crystalline solid. A small sample was recrystallized from dichloromethane to give pale yellow crystals, mp 59-61 C. IR (soln) 3050 and $3020(\mathrm{C}=\mathrm{C}-\mathrm{H}), 2940(\mathrm{C}-\mathrm{H}), 2100\left(\mathrm{C}-\mathrm{HN}_{2}\right)$, $1635(\mathrm{C}=\mathrm{O}), 1580(\mathrm{ArC}=\mathrm{C}), 1580(\mathrm{C}=\mathrm{N}), 1455$ and $1440(\mathrm{~N}=\mathrm{N}) \mathrm{cm}^{-1} .{ }^{1} \mathrm{H}$ NMR $\delta 7.35(1 \mathrm{H}, \mathrm{d}, J$ $=7.4 \mathrm{~Hz}, \mathrm{H}-6), 7.15(1 \mathrm{H}, \mathrm{t}, J=7.4 \mathrm{~Hz}, \mathrm{H}-7), 7.07(1 \mathrm{H}, \mathrm{d}, J=7.4 \mathrm{~Hz}, \mathrm{H}-8), 6.93(1 \mathrm{H}, \mathrm{dd}, J=$ 17.4, $11.0 \mathrm{~Hz}, \mathrm{H}-5 \mathrm{a}), 5.63$ (1H, dd, $J=17.4,1.5 \mathrm{~Hz}, \mathrm{H}-5 \mathrm{~b}$-trans), 5.40 (1H, br s, $\left.\mathrm{CHN}_{2}\right), 5.32$ (1H, dd, $J=11.0,1.5 \mathrm{~Hz}, \mathrm{H}-5 \mathrm{~b}-$-cis), 3.08-2.89 (3H, m, H-1 and H-4), 2.80-2.64 (2H, m, H-2 and H-4), 2.21-2.15 (1H, m, H-3), 1.92-1.82 (1H, m, H-3). ${ }^{13} \mathrm{C}$ NMR $\delta 197.2(\mathrm{C}=\mathrm{O}), 136.9$ (C5), 135.1 (C-8a), 134.5 (C-5a), 132.9 (C-4a), 128.7 (C8), 125.8 (C-6), 123.5 (C-7), 115.8 (C5b), $53.8\left(\mathrm{CHN}_{2}\right), 45.1$ (C-2), 32.3, 26.2 and $26.0\left(3 \times \mathrm{CH}_{2}\right)$. LRMS $226\left(\mathrm{M}^{+}, 3 \%\right), 198\left(\mathrm{M}-\mathrm{N}_{2}\right.$, 26\%), 183 (23\%), 170 (39), 155 (85), 141 (90), 128 (95), 115 (100). HRMS: found $\left(\mathrm{M}^{+}\right)$: 226.1105; $\mathrm{C}_{14} \mathrm{H}_{14} \mathrm{~N}_{2} \mathrm{O}$ requires 226.1106. Anal. Calcd for $\mathrm{C}_{14} \mathrm{H}_{14} \mathrm{~N}_{2} \mathrm{O}: \mathrm{C}, 74.31 ; \mathrm{H}, 6.24 ; \mathrm{N}$, 12.38. Found: C, 74.71; H, 6.40; N, 11.98 .

8-Vinyl-2,3-dihydro-1H-3,4b-methanocyclopropa-[1,2:1,3]-dibenzene-4-(4a-H)-one (22). To a suspension of $\mathrm{Cu}(\mathrm{acac})_{2}(2 \mathrm{mg}, 2 \mathrm{~mol} \%)$ in 1,2-dichloroethane $(1 \mathrm{~mL})$ at reflux was added dropwise via syringe pump a solution of diazoketone (46 mg, $0.2 \mathrm{mmol}$ ) in 1,2-dichloroethane ( $3 \mathrm{~mL}$ ) over 1.5 hours. Complete consumption of the starting material was observed and the reaction allowed to cool to room temperature. Removal of the solvent under reduced pressure, followed by purification using column chromatography $(10 \%$ ethyl acetate/petrol) afforded the major product $22(12 \mathrm{mg}, 32 \%)$ as a yellow oil. ${ }^{1} \mathrm{H}$ NMR $\delta 6.52(1 \mathrm{H}, \mathrm{dd}, J=17.4,11.0 \mathrm{~Hz}, \mathrm{H}-$ 10), $6.18(1 \mathrm{H}, \mathrm{d}, J=13.7 \mathrm{~Hz}, \mathrm{H}-5$ or H-7), $6.15(1 \mathrm{H}, \mathrm{d}, J=10.7 \mathrm{~Hz}, \mathrm{H}-5$ or H-7), 6.02 (1H, m, H-6), 5.48 (1H, d, $J=17.4 \mathrm{~Hz}, \mathrm{H}-10 \mathrm{a}-$ trans), 5.14 (1H, d, $J=11.0 \mathrm{~Hz}, \mathrm{H}-10 \mathrm{a}-$-cis $), 2.56-2.48$ (2H, m, H-9ß, H-1), 2.27-2.25 (1H, m, H-3), 2.59-2.48 (1H, m, H-1), $2.52(1 \mathrm{H}, \mathrm{d}, J=12.0 \mathrm{~Hz}$, $\mathrm{H}-9 \alpha), 2.47-1.81(2 \mathrm{H}, \mathrm{m}, \mathrm{H}-2), 1.78(1 \mathrm{H}, \mathrm{s}, \mathrm{H}-4 \mathrm{a}) .{ }^{13} \mathrm{C} \mathrm{NMR} \delta 216.6(\mathrm{C}=\mathrm{O}), 140.0\left(\mathrm{CH}_{2}=\mathrm{CH}\right)$, 134.9 (C-8), 129.8, 122.7 and 119.7 (C-7, C-6 and C-5), $115.7\left(\mathrm{CH}_{2}=\mathrm{CH}\right), 46.9(\mathrm{C}-8 \mathrm{a}), 44.6(\mathrm{C}-$ 4b), 41.7 (C-3), 32.6 (C-4a), 30.7, 27.8 and $20.3\left(3 \times \mathrm{CH}_{2}\right)$.

Methyl (3-RS,4a-SR,4b-RS,6a-SR,7-RS,10b-RS)-2,3,6a,7,8,9-hexahydro-1H-3,4b-methanobenzo[1,3]-cyclopropa-[1,2-a]naphthalene-4-(4aH)-one-7-carboxylate (24). To a suspension of $\mathrm{Cu}(\mathrm{acac})_{2}(5 \mathrm{mg}, 2 \mathrm{~mol} \%)$, in dichloroethane $(5 \mathrm{~mL})$ at reflux, was added dropwise via syringe pump a solution of the diazoketone $(200 \mathrm{mg}, 0.89 \mathrm{mmol})$ in dichloroethane $(15 \mathrm{~mL})$ over 1.5 hours. Complete consumption of the starting material was observed within 2 hours. Methyl acrylate $(1.6 \mathrm{~mL}, 20$ equiv.) was added and the solution heated to reflux for a further 20 hours, after which time the reaction was cooled to room temperature. Removal of the solvent in vacuo, followed by purification using column chromatography $(20 \%$ ethyl acetate/petrol), afforded a mixture of three of the diastereomeric adducts $(65 \mathrm{mg}, 26 \%)$ in the ratio 4:2:1. The major isomer 24 was separated using MPLC. IR (soln) 2950 and $2870(\mathrm{C}-\mathrm{H}), 1730(\mathrm{C}=\mathrm{O}), \mathrm{cm}^{-1}$. ${ }^{1} \mathrm{H}$ NMR $\delta$ $5.85(1 \mathrm{H}, \mathrm{dd}, J=9.6,1.7 \mathrm{~Hz}, \mathrm{H}-6), 5.61(1 \mathrm{H}, \mathrm{m}, \mathrm{H}-1), 5.41(1 \mathrm{H}, \mathrm{dd}, J=9.6,1.7 \mathrm{~Hz}, \mathrm{H}-7), 3.65$ $\left(3 \mathrm{H}, \mathrm{s}, \mathrm{OCH}_{3}\right), 2.89-2.84(2 \mathrm{H}, \mathrm{m}, \mathrm{H}-4$ and H-5), 2.39 (1H, s, H-15), 2.31-1.82 (10H, m), 1.99 $(1 \mathrm{H}, \mathrm{d}, J=11.7 \mathrm{~Hz}, \mathrm{H}-14 \alpha) .{ }^{13} \mathrm{C} \mathrm{NMR} \delta 213.4(\mathrm{C} 16), 173.8(\mathrm{C}=\mathrm{O}), 133.1(\mathrm{C} 10), 127.5,127.3$ and $121.3(\mathrm{C} 1, \mathrm{C} 6$ and $\mathrm{C} 7), 51.4\left(\mathrm{OCH}_{3}\right), 43.9,43.2$ and $41.9(\mathrm{C} 4, \mathrm{C} 5$ and $\mathrm{C} 13), 40.8$ and 38.9 
(C8 and C9), 35.2 (C15), 32.4, 28.0, 24.1, 23.1 and $19.0\left(5 \times \mathrm{CH}_{2}\right)$. LRMS (M+, 10\%), 239 (32), $225\left(\mathrm{M}-\mathrm{CO}_{2} \mathrm{CH}_{3}, 12\right), 197$ (retro-DA, 8), 180 (14), 169 (8), 152 (8), 141 (18), 132 (25), 84 (100). HRMS: found $\left(\mathrm{M}^{+}\right) 284.1413 ; \mathrm{C}_{18} \mathrm{H}_{20} \mathrm{O}_{3}$ requires 284.1412 .

Methyl (3-RS,4a-SR,4b-RS,6a-SR,7-SR,10b-RS)-2,3,6a,7,8,9-7-chlorohexahydro-1H-3,4bmethano benzo-[1,3]-cyclopropa[1,2-a]naphthalene-4-(4aH),5(6H)-dione-7-carboxylate. To a suspension of $\mathrm{Cu}(\mathrm{acac})_{2}(1 \mathrm{mg}, 2 \mathrm{~mol} \%)$, in dichloroethane $(1 \mathrm{~mL})$ at reflux, was added dropwise via syringe pump a solution of ( \pm )-diazomethyl 8-methoxy-5-vinyl-1,2,3,4tetrahydronaphthalen-2-yl ketone $(40 \mathrm{mg}, 0.16 \mathrm{mmol})$ in dichloroethane $(4 \mathrm{~mL})$ over the course of 1 hour. Complete consumption of the starting material was observed within 1.5 hours. Methyl 2-chloropropenoate $(0.1 \mathrm{~mL}, 1.1 \mathrm{mmol}, 7$ equiv. $)$ and 1,2-epoxybutane $(0.2 \mathrm{~mL})$ were added and the solution heated at reflux for a further 20 hours, after which the reaction was cooled to room temperature. Removal of the solvent in vacuo followed by purification using column chromatography (20\% ethyl acetate/petrol) afforded a crude mixture of the four isomeric adducts, in the ratio 10:2:2:1. Further column chromatography isolated the major isomer as a pale yellow solid, which was determined to be the 5-one derived from hydrolysis of the enol ether 25 (35mg, 65\%); IR (soln) $3030(\mathrm{C}=\mathrm{C}-\mathrm{H}), 2960(\mathrm{C}-\mathrm{H}), 1740(\mathrm{OC}=\mathrm{O}), 1690(\mathrm{RC}=\mathrm{O})$ $\mathrm{cm}^{-1}$; ${ }^{1} \mathrm{H}$ NMR $\delta 5.79(1 \mathrm{H}, \mathrm{m}, \mathrm{H}-1), 3.81\left(3 \mathrm{H}, \mathrm{s}, \mathrm{OCH}_{3}\right), 3.02(1 \mathrm{H}, \mathrm{s}, \mathrm{H}-15), 2.92(1 \mathrm{H}, \mathrm{dd}, J=$ $12.7,6.0 \mathrm{~Hz}, \mathrm{H}-14 \beta), 2.70-2.40$ (4H, m), 2.35-2.10 (5H, m), 2.05-1.85 (3H, m), 1.81 (1H, d, $J$ $=12.7 \mathrm{~Hz}, \mathrm{H}-14 \alpha) .{ }^{13} \mathrm{C}$ NMR $\delta 210.1(\mathrm{C} 16), 209.7(\mathrm{C} 7), 169.6(\mathrm{C}=\mathrm{O}), 131.0(\mathrm{C} 10), 121.7(\mathrm{C} 1)$, $68.4(\mathrm{C} 4), 53.0\left(\mathrm{OCH}_{3}\right), 47.8$ and $44.9(\mathrm{C} 8$ and $\mathrm{C} 9), 40.6,40.5$ and $38.6(\mathrm{C} 5, \mathrm{C} 13$ and $\mathrm{C} 15)$, 29.5, 27.3, 26.9, 26.5, 22.2 and $18.4\left(6 \times \mathrm{CH}_{2}\right)$. LRMS $334\left(\mathrm{M}^{+}, 92 \%\right), 298(\mathrm{M}-\mathrm{Cl}, 62), 267(\mathrm{M}-$ $\left.\mathrm{Cl}, \mathrm{OCH}_{3}, 98\right), 239\left(\mathrm{M}-\mathrm{Cl}, \mathrm{CO}_{2} \mathrm{CH}_{3}, 89\right), 214$ (66), 128 (64), 91 (100). HRMS: found $\left(\mathrm{M}^{+}\right)$ $334.0971 ; \mathrm{C}_{18} \mathrm{H}_{19} \mathrm{ClO}_{4}$ requires 334.0972.

Methyl (3-RS,4a-SR,4b-RS,6a-SR,7-SR,10b-RS)-7-chloro-2,3,6a,7,8,9-hexahydro-1H-3,4bmethanobenzo-[1,3]-cyclopropa-[1,2-a]-naphthalene-4(4a-H)one-7-carboxylate (26). To a suspension of $\mathrm{Cu}(\mathrm{acac})_{2}(2.5 \mathrm{mg}, 2 \mathrm{~mol} \%)$, in dichloroethane $(2 \mathrm{~mL})$ at reflux, was added dropwise via syringe pump a solution of the diazoketone $(100 \mathrm{mg}, 0.45 \mathrm{mmol})$ in dichloroethane $(8 \mathrm{~mL})$ over the course of 1 hour. Complete consumption of the starting material was observed within 1.5 hours. Methyl 2-chloropropenoate $(0.2 \mathrm{~mL}, 2.2 \mathrm{mmol}, 5$ equiv.) was added and the solution heated to reflux for a further 20 hours, after which time the reaction was cooled to room temperature. Removal of the solvent in vacuo followed by purification using column chromatography (2\% methanol/dichloromethane) afforded a crude mixture of three of the isomeric adducts. The major isomer 26 was separated by further column chromatography (20 mg, 15\%). ${ }^{1} \mathrm{H}$ NMR $\delta 5.93(1 \mathrm{H}, \mathrm{dd}, J=9.8,3.2 \mathrm{~Hz}, \mathrm{H}-6), 5.81(1 \mathrm{H}, \mathrm{dd}, J=9.8,2.2 \mathrm{~Hz}, \mathrm{H}-$ 7), 5.64-5.59 (1H, m, H-10), $3.76\left(3 \mathrm{H}, \mathrm{s}, \mathrm{OCH}_{3}\right), 3.13-3.08(1 \mathrm{H}, \mathrm{m}, \mathrm{H}-5), 2.45-2.18(7 \mathrm{H}, \mathrm{m})$, 2.11-1.90 (3H, m) $2.05(1 \mathrm{H}, \mathrm{s}, \mathrm{H}-15), 2.04(1 \mathrm{H}, \mathrm{d}, J=11.7 \mathrm{~Hz}, \mathrm{H}-14 \alpha) .{ }^{13} \mathrm{C}$ NMR $\delta 213.4$ (C16), $169.3(\mathrm{C}=\mathrm{O}), 132.4(\mathrm{C} 10), 126.0,125.1$ and $120.3(\mathrm{C} 1, \mathrm{C} 6$ and $\mathrm{C} 7), 69.7(\mathrm{C} 4), 52.8$ $\left(\mathrm{OCH}_{3}\right), 45.5$ and 41.9 (C13 and $\left.\mathrm{C} 5\right), 43.4$ and 38.4 (C8 and $\left.\mathrm{C} 9\right), 34.4$ (C15), 31.5, 28.0, 24.3, 22.6, and $19.3\left(5 \times \mathrm{CH}_{2}\right)$. 
3-RS,4a-SR,4b-RS,6a-SR,7-RS,8-RS,10b-RS-2,3,6a,7,8,9-Hexahydro-1H-3,4b-methanobenzo [1,3]-cyclopropa-[1,2-a]-naphthalene-4-(4aH)-one-7,8-dicarboxylic anhydride (28). To a suspension of $\mathrm{Cu}(\mathrm{acac})_{2}(5 \mathrm{mg}, 2 \mathrm{~mol} \%)$, in dichloroethane $(5 \mathrm{~mL})$ at reflux, was added dropwise via syringe pump a solution of the diazoketone $(200 \mathrm{mg}, 0.89 \mathrm{mmol})$ in dichloroethane $(15 \mathrm{~mL})$ over the course of 1.5 hours. Complete consumption of the starting material was observed within 2 hours. Maleic anhydride (400 mg, $4 \mathrm{mmol}, 4.5$ equiv.) was added and the solution heated to reflux for a further 6 hours, after which time the reaction was cooled to room temperature. Removal of the solvent in vacuo followed by purification using column chromatography (50\% ethyl acetate/petrol) afforded the major product $28(140 \mathrm{mg}, 53 \%)$ as a white solid. A small sample was recrystallized from hot acetone to give white needles, mp 240-242 C. ${ }^{1} \mathrm{H}$ NMR $\left(\right.$ DMSO-d $\left._{6}\right) \delta 6.19-6.16(1 \mathrm{H}, \mathrm{m}, \mathrm{H}-1), 6.05$ (1H, dd, $J=10.1,2.3 \mathrm{~Hz}, \mathrm{H}-6$ or H-7), 5.73 (1H, dd, $J=10.1,2.3 \mathrm{~Hz}, \mathrm{H}-6$ or H-7), 3.66-3.55 (2H, m, H-3 and H-4), 2.90-2.88 (1H, m, H-5), 2.64-

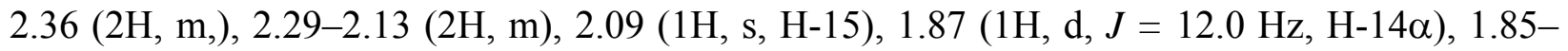
$1.62(4 \mathrm{H}, \mathrm{m}) .{ }^{13} \mathrm{C}$ NMR $\left(\mathrm{DMSO}_{-} \mathrm{d}_{6}\right) \delta 211.3(\mathrm{C} 16), 175.8$ and $172.0(2 \times \mathrm{C}=\mathrm{O}), 138.1(\mathrm{C} 10)$, 126.4, 124.9, 123.2 (C1, C6 and C7), 45.8 (C4), 45.7 (C3), 41.2 (C15), 40.8 (C13), 38.8 and 36.1 (C8 and C9), 33.9 (C5), 32.0, 27.5, 24.7 and $19.2\left(4 \times \mathrm{CH}_{2}\right)$. LRMS (M+, 27\%), $268(\mathrm{M}-\mathrm{CO}$, 100), $223\left(\mathrm{M}-\mathrm{CO}_{2} \mathrm{H}, \mathrm{CO}, 87\right), 198$ (retro-Diels-Alder, 30), $195\left(\mathrm{M}-\mathrm{CO}_{2} \mathrm{H}, 2 \times \mathrm{CO}, 93\right)$. HRMS: found $\left(\mathrm{M}^{+}\right)$296.1048; $\mathrm{C}_{18} \mathrm{H}_{16} \mathrm{O}_{4}$ requires 296.1049.

\section{3-RS,4a-SR,4b-RS,6a-SR,7-RS,8-RS,10b-RS-7-Methyl-2,3,6a,7,8,9-hexahydro-1H-3,4b-} methano-benzo-[1,3]-cyclopropa-[1,2-a]-naphthalene-4-(4a-H)-one-7,8-dicarboxylic anhydride (29). To a suspension of $\mathrm{Cu}(\mathrm{acac})_{2}(12 \mathrm{mg}, 2 \mathrm{~mol} \%)$, in dichloroethane $(10 \mathrm{~mL})$ at reflux, was added dropwise via syringe pump a solution of diazoketone (552 $\mathrm{mg}, 2.45 \mathrm{mmol}$ ) in dichloroethane $(40 \mathrm{~mL})$ over the course of 2.5 hours. Complete consumption of the starting material was observed within 3 hours, after which time citraconic anhydride ( $0.5 \mathrm{~mL}, 3$ equiv.) was added and the solution heated at reflux for a further 18 hours. Removal of the solvent in vacuo followed by purification using column chromatography (40\% ethyl acetate/petrol) afforded 29 plus a minor isomer in the ratio 8:1, (300 $\mathrm{mg}, 40 \%)$, as a cream solid. A small sample was recrystallized from chloroform to give white needles of the major isomer, mp 182184 C. IR (soln) $3020(\mathrm{C}=\mathrm{C}-\mathrm{H}), 2900$ and $2750(\mathrm{C}-\mathrm{H}), 1780(\mathrm{OC}=\mathrm{O}), 1720(\mathrm{RC}=\mathrm{O}) \mathrm{cm}^{-1} \cdot{ }^{1} \mathrm{H}$ NMR (COSY) $\delta 6.22(1 \mathrm{H}, \mathrm{m}, \mathrm{H}-1), 6.82(1 \mathrm{H}, \mathrm{dd}, J=10.3,2.7 \mathrm{~Hz}, \mathrm{H}-6), 5.82(1 \mathrm{H}, \mathrm{ddd}, J=$ 10.3, 3.1, 1.1 Hz, H-7), 3.04 (1H, dd, $J=6.0,2.1 \mathrm{~Hz}, \mathrm{H}-3), 2.82(1 \mathrm{H}$, ddd, $J=15.3,7.7,2.3 \mathrm{~Hz}$, H-2), 2.49-2.47 (1H, m, H-5), 2.43-2.35 (1H, m, H-11), 2.29 (1H, ddd, J = 11.7, 5.8, 1.8 Hz, H$14 \beta$ ), 2.24-2.16 (2H, m, H-2 and H-13), 1.97 (1-H, d, $J=11.7 \mathrm{~Hz}, \mathrm{H}-14 \alpha), 1.94-1.82$ (3H, m, $\mathrm{H}-11$ and $2 \times \mathrm{H}-12), 1.58\left(3 \mathrm{H}, \mathrm{s}, \mathrm{CH}_{3}\right), 1.49(1 \mathrm{H}, \mathrm{s}, \mathrm{H}-15) .{ }^{13} \mathrm{C}$ NMR (HETCOR) $\delta 212.4(\mathrm{C} 16)$, 173.6 and $172.8(2 \times \mathrm{C}=\mathrm{O}), 137.8$ (C10), 128.9 (C6), $125.6(\mathrm{C} 1), 121.0(\mathrm{C} 7), 50.5(\mathrm{C} 4), 49.3$ (C3), 46.6 (C5), 41.6 (C15), 40.9 (C13), 39.9 and 34.5 (C8 and C9), 32.6 (C14), 27.8 (C12), 24.1 (C2), $22.1\left(\mathrm{CH}_{3}\right), 20.1(\mathrm{C} 11)$. LRMS $310\left(\mathrm{M}^{+}, 10 \%\right), 282(\mathrm{M}-\mathrm{CO}, 68), 237\left(\mathrm{M}-\mathrm{CO}, \mathrm{CO}_{2} \mathrm{H}, 63\right)$, $209\left(\mathrm{M}-\mathrm{CO}_{2} \mathrm{H}, 2 \times \mathrm{CO}, 60\right), 198$ (retro Diels-Alder, 93), 155 (100). HRMS: found $\left(\mathrm{M}^{+}\right)$ $310.1205 ; \mathrm{C}_{19} \mathrm{H}_{18} \mathrm{O}_{4}$ requires 310.1205 . 


\section{References}

1. King, G. R.; Mander, L. N.; Monck, N. J. T.; Morris, J. C.; Zhang, H. J. Am. Chem. Soc. 1997, 119, 3828.

2. Morris, J. C.; Mander, L. N.; Hockless, D. C. R. Synthesis 1998, 455.

3. Dev, S.; Misra, R. CRC Handbook of Terpenoids, Diterpenoids Vol. IV: Tetracyclic and Pentacyclic Diterpenoids; CRC Press: Boca Raton, Florida, 1986.

4. Adams, R.; Ulich, H. J. Am. Chem. Soc. 1920, 42, 599.

5. Mander, L. N.; Pyne, S. G. J. Am. Chem. Soc. 1979, 101, 3373. (b) Mander, L. N.; Pyne, S. G. Aust. J. Chem. 1981, 34, 1899.

6. Kaplan, F.; Melroy, G. K. J. Am. Chem. Soc. 1966, 88, 950.

7. Lombardo, L. Tetrahedron Lett. 1982, 23, 4293.

8. Tour, J. M.; Bedworth, P. V.; Wu, R. Tetrahedron Lett. 1989, 30, 3927.

9. Peterson, D. J. J. Org. Chem. 1968, 33, 780.

10. Freerksen, R. W.; Selikson, S. J.; Wroble, R. R.; Kyler, K. S.; Watt, D. S. J. Org. Chem. 1987, 48, 4080 .

11. Boutagy, J.; Thomas, R. Chem. Rev. 1974, 74, 87.

12. (a) Pettit, G. R.; Knight, J. C.; Herald, C. L. J. Org. Chem. 1970, 35, 1790. (b) Dugger, R. W.; Heathcock, C. H. Synth. Commun. 1980, 10, 509.

13. Echavarren, A. M.; Stille, J. K. J. Am. Chem. Soc. 1987, 109, 5478.

14. (a) Buisson, D.; Cecchi, R.; Laffitte, J.-A.; Guzzi, U.; Azerad, R. Tetrahedron Lett. 1994, 35, 3091. (b) Genêt, J. P.; Pfister, X.; Ratovelomanana-Vidal, V.; Pinel, C.; Laffitte, J. A. Tetrahedron Lett. 1994, 35, 4559. (c) Mander, L. N.; Morris, J. C. J. Org. Chem. 1997, 62, 749.

15. Still, W. C.; Kahn, M.; Mitra, A. J. Org. Chem. 1978, 43, 2923.

16. Johnson, D.W.; Mander, L. N. Aust. J. Chem. 1974, 27, 1277. 